Journal of Fluid Mechanics

http://journals.cambridge.org/FLM

Journal of

Additional services for Journal of Fluid Mechanics:

Email alerts: $\underline{\text { Click here }}$

Subscriptions: $\underline{\text { Click here }}$

Commercial reprints: $\underline{\text { Click here }}$

Terms of use : $\underline{\text { Click here }}$

\title{
Dynamics of monopolar vortices on a topographic beta-plane
}

\author{
J.-B. FL\&Oacute;R and I. EAMES \\ Journal of Fluid Mechanics / Volume 456 / April 2002, pp 353 - 376 \\ DOI: 10.1017/S0022112001007728, Published online: 09 April 2002
}

Link to this article: http://journals.cambridge.org/abstract_S0022112001007728

How to cite this article:

J.-B. FLÓR and I. EAMES (2002). Dynamics of monopolar vortices on a topographic beta-plane. Journal of Fluid Mechanics, 456, pp 353-376 doi:10.1017/S0022112001007728

Request Permissions : $\underline{\text { Click here }}$ 


\title{
Dynamics of monopolar vortices on a topographic beta-plane
}

\author{
By J.-B. FLÓR AND I. EAMES \\ LEGI, Laboratoires des Ecoulements Geophysiques et Industriels BP 53, \\ 38041 Grenoble Cedex 09, France \\ Department of Mechanical Engineering, University College London, Torrington Place, \\ London, WC1E 7JE, UK
}

(Received 8 April 2000 and in revised form 11 September 2001)

The dynamics of a cyclonic monopolar vortex on a topographic beta-plane are studied experimentally and theoretically. Detailed measurements of the vortex structure are conducted using high-resolution quantitative velocity measurements. The initial velocity profiles were described in terms of a radius $R_{v m}$, maximum azimuthal velocity $v_{\theta m}$, and a dimensionless parameter $\alpha$ which characterizes the steepness of the velocity profile. The initial direction of motion of the monopolar vortex is critically dependent on $\alpha$ and weakly dependent of the initial strength and size of the vortex: isolated vortices $(\alpha \sim 3)$ move north, whereas non-isolated vortices characterized by $\alpha \sim 1$ move northwest. When the azimuthal velocity decays slowly with radial distance $(\alpha<1.4)$, Rossby wave generation dominates the vortex dynamics and the translational speed of the vortex correlates with the Rossby wave speed. When the azimuthal velocity decays rapidly with radial distance $(\alpha>1.4)$ the vortex is isolated and the translational speed is much slower than the Rossby wave speed. To interpret the effect of the vortex structure on the direction of motion, a mechanistic model is developed which includes the Rossby force and a lift force arising from circulation around the vortex, but does not include the effect of Rossby waves. The Rossby force results from the integrated effect of the Coriolis force on the vortex and drives the vortex north; the lift force is determined from the circulation around the vortex and drives the vortex west. Comparison with the experimental data reveals two regimes: $\alpha<1.4$, where the vortex dynamics are dominated by Rossby waves whereas for $\alpha>1.4$ Rossby waves are weak and favourable agreement is found with the mechanistic model.

\section{Introduction}

The propagation of monopolar vortices in the presence of a vorticity gradient has been a subject of wide interest. In geophysical flows, vortices such as hurricanes, tropical cyclones in the atmosphere or meso-scale eddies in the ocean are known to translate over large distances owing to the variation of the planetary Coriolis force. The self-induced propagation of vortices plays an important role in the transport of mass, heat and momentum over large distances. Experimental, theoretical and numerical studies have shown conclusively that cyclonic monopolar vortices tend to move towards the northwest direction (e.g. van Heijst 1994). Although there has been a great deal of progress in interpreting and modelling the dynamics of monopolar vortices on the beta-plane, issues such as which parameters control their direction of translation, speed and characteristic structure are still not fully understood. The aim 
of this paper is to resolve some of these issues through a systematic experimental study over a wide range of parameters and to compare these results with a mechanistic model, based on potential flow theory.

Rossby (1948) originally explained the drift of monopolar vortices on a beta-plane by the integrated effect of the Coriolis force over the vortex which results in a net force to the north, later referred to as the Rossby force. The effect of fluid advection by the vortex, which was not taken into account by Rossby (1948), is to generate positive vorticity at its west (by displacing fluid north) and negative vorticity at its east (by displacing fluid south), leading to a westward shift of the vorticity maximum (Flierl et al. 1980). In addition, the nonlinear interaction of vorticity generated outside the vortex leads to a dipolar component which transports the vortex centre perpendicular to the direction of the shift (Stern \& Radko 1998). As has been demonstrated in various experimental and numerical simulations, most vortices do propagate to the northwest.

Reznik (1992) examined the motion of singular vortices on the beta-plane and focused on the distortion of the ambient potential vorticity field by the vortical flow. The intensity of the vortices was characterized by $\delta$, the ratio between the turnover time scale within the vortex and the time period of oscillation of long Rossby waves. Intense vortices $(\delta \gg 1)$ were found to move initially northward, and ultimately tend to an almost zonal velocity close to the Rossby wave speed. Intense vortices wrap around ambient (oppositely signed) vorticity and eventually become isolated. Moderate vortices $(\delta \geqslant 1)$ were found to be more complicated because of their interaction with Rossby waves. These vortices do not show a dipolar motion alternating in westward and northward directions which is observed for intense vortices (see Reznik 1992; Korotaev \& Fedotov 1994) and thus behave differently. Calculation of the change of the vortical field and induced velocity at the vortex centre, permitted the vortex trajectory to be determined. This trajectory is unique because the vortex area is infinitesimally small.

Reznik \& Dewar (1994) further developed Reznik's (1992) analysis by considering the dynamics of intense barotropic vortices of arbitrary initial velocity field, including isolated vortices. Llewellyn Smith (1997) also examined intense non-isolated vortices, and focused on the connection between the near-field and the far-field flowdominated by Rossby waves - using matched asymptotic analysis. A weakness in the predictive power of these models is their failure to predict uniquely the vortex trajectory because its centre can be identified with either the relative vorticity maximum or the maximum of the streamfunction.

Sutyrin \& Flierl (1994) showed that the direction of translation depends on the relative size of the vortex to the deformation radius. When the vortex is much smaller than the deformation radius, it initially moves northward, whereas axisymmetrization due to the differential rotation and advection of vorticity leads to a gradual westward motion in agreement with Reznik (1992). However, when the vortex is comparable to the deformation radius, the initial meridional motion is negligible, as confirmed by experimental measurements by Stegner \& Zeitlin (1998) for low-Rossby-number vortices. Stegner \& Zeitlin (1998) suggest a quasi-geostrophic regime with a vortex scale smaller than the deformation radius, and an ageostrophic regime represented by a vortex scale larger than the deformation radius.

Despite the large number of analytical and numerical studies on monopolar vortices moving on the beta-plane there are comparatively few laboratory investigations. In most laboratory experiments the vorticity gradient is modelled by a topographic betaplane consisting of a sloping bottom in a tank rotating at a constant angular velocity. 


\begin{tabular}{|c|c|c|c|c|c|c|c|}
\hline Geometry and size & Mean depth $H_{0}(\mathrm{~m})$ & slope $\frac{\mathrm{d} H}{\mathrm{~d} y}$ & $\Omega\left(\mathrm{rad} \mathrm{s}^{-1}\right)$ & $\beta\left(\mathrm{m}^{-1} \mathrm{~s}^{-1}\right)$ & Ro & $\delta$ & $B u^{1 / 2}$ \\
\hline $\begin{array}{l}\text { Present experiments: } \\
\text { square }(0.96 \mathrm{~m} \times 0.96 \mathrm{~m}) \\
\text { circular }(\text { diameter } 13.0 \mathrm{~m})\end{array}$ & $\begin{array}{l}0.182 \\
0.35\end{array}$ & $\begin{array}{l}5 / 96 \text { (slope) } \\
16 / 300 \text { (slope) }\end{array}$ & $\begin{array}{l}0.5 \\
0.125\end{array}$ & $\begin{array}{l}0.289 \\
0.0406\end{array}$ & $\begin{array}{l}0.1-0.8 \\
0.2-1.2\end{array}$ & $\begin{array}{l}8-107 \\
12-64\end{array}$ & $\begin{array}{l}0.012-0.04 \\
0.012-0.018\end{array}$ \\
\hline $\begin{array}{l}\text { Carnevale et al. }(1991): \\
\quad \text { circular (diameter 0.925 m) }\end{array}$ & $\begin{array}{l}0.171 \\
0.171,0.165\end{array}$ & $\begin{array}{l}\text { 10/75 (slope) } \\
5 / 46 \text { (cone) }\end{array}$ & $\begin{array}{l}0.6 \\
0.6,1.0\end{array}$ & $\begin{array}{l}0.914 \\
0.76,1.32\end{array}$ & $\begin{array}{l}O(1) \\
O(1)\end{array}$ & $O(10)$ & $\begin{array}{l}0.1-0.3 \\
O(0.1)\end{array}$ \\
\hline $\begin{array}{l}\text { Firing \& Beardsley (1976): } \\
\text { circular (diameter } 0.279 \mathrm{~m} \text { ) } \\
\text { rigid lid }\end{array}$ & 0.08 & $2.79 / 27.9$ & 6.28 & 15.7 & $0.02-0.15$ & $0.81-6.1$ & 0 \\
\hline $\begin{array}{l}\text { Masuda et al. }(1990): \\
\quad \text { square }(0.508 \mathrm{~m} \times 0.508 \mathrm{~m})\end{array}$ & 0.24 & $16.9 / 50.8$ & 1.99 & 6.81 & 0.4 & 4.7 & 0.008 \\
\hline $\begin{array}{l}\text { Stegner \& Zeitlin (1998): } \\
\text { parabolic }(0.45 \mathrm{~m}) \\
z=2.67 r^{2}\end{array}$ & $4-24 \mathrm{~mm}$ & & $\begin{array}{l}7.43 \\
\text { (mean) }\end{array}$ & $\approx 0.1$ & $0.1-0.3$ & $\approx 2-5$ & $0.8-4.2$ \\
\hline $\begin{array}{l}\text { TABLE 1. Parameters of relevan } \\
\text { three concern the vortex dynat } \\
\text { phase speed of Rossby waves, } \\
\text { The strength of the beta-plane } \\
\text { For the parabolic vessel } \beta=R\end{array}$ & $\begin{array}{l}\text { otating tank experime } \\
\text { cs, with } R o=v_{\theta m} / f R_{v} \\
\text { d the Burgers numbe } \\
\text { characterized by } \beta=2 \\
\Omega^{2} \cos \theta \sin \theta / g .\end{array}$ & $\begin{array}{l}\text { on } \beta \text {-plane mor } \\
\text { he Rossby numb } \\
3 u=\left(R_{v m} / R_{d}\right)^{2} \mathrm{~d} \\
(\mathrm{~d} H / \mathrm{d} y) / H \text { wher }\end{array}$ & $\begin{array}{l}\text { olar vortices. } \\
\delta=v_{\theta m} / \beta R_{v n}^{2} \\
\text { ned as the rat } \\
H \text { is the mean }\end{array}$ & $\begin{array}{l}\text { te first } 5 \text { para } \\
\text { the ratio of } \\
\text { of vortex rad } \\
\text { ter depth cor }\end{array}$ & $\begin{array}{l}\text { ters are bac } \\
\text { rtex maxim } \\
\text { to the def } \\
\text { ted for the }\end{array}$ & $\begin{array}{l}\text { round con } \\
\text { l velocity } \\
\text { nation sca } \\
\text { face defor }\end{array}$ & $\begin{array}{l}\text { ints, the latter } \\
\text { mpared to the } \\
R_{d}=\sqrt{g H} / f \\
\text { tion } \Omega^{2} r^{2} / 2 g \text {. }\end{array}$ \\
\hline
\end{tabular}


Table 1 summarizes the relevant experimental studies. The physical analogy between a (planetary) beta-plane, characterized by a gradient of the Coriolis parameter, and a topographic beta-plane is based on the dynamical equivalence between the displacement of fluid and the production of potential vorticity. Carnevale, Kloosterziel \& van Heijst (1991) studied the dynamics of monopolar vortices on a topographic beta-plane (generated by a slope and a cone) and comparisons with numerical simulations for the motion of the vortex centre and transport of dye showed good agreement. Only two types of vortex were studied by Carnevale et al. (1991): a quasi non-isolated vortex (generated by a sink) and an isolated vortex (generated by stirring the fluid), which were observed to move to the northwest and north, respectively, at least during an initial stage of the flow evolution. It is also relevant to mention the more recent experimental study on monopolar vortices in a thin layer of fluid on a rotating parabolic dish by Stegner \& Zeitlin (1998), who varied the relative size of the vortex to the deformation radius.

The influence of the beta-plane and free-surface deformation on the generation of potential vorticity may be understood from the vorticity equation. The nondimensional potential vorticity is defined by

$$
\Pi=\frac{f}{H_{0}}\left[1+R o\left(\omega-B u \eta+\eta_{b}\right)\right],
$$

with $\eta_{b}$ the beta-effect due to the inclined bottom and $\eta$ the surface deformation. The leading-order non-dimensional vorticity equation becomes

$$
\frac{\mathrm{D}}{\mathrm{D} t}[\omega-B u \eta]+\frac{1}{\delta} u_{y}=0,
$$

which is derived in the limit of small Rossby number $R o=v_{\theta m} / f R_{v m}$, with $f$ being twice the rotation rate, and $R_{v m}, v_{\theta m}$ the vortex radius and maximum azimuthal velocity, respectively (see Pedlosky 1979 p. 92). The Burgers number $B u=\left(R_{v m} / R_{d}\right)^{2}$ is defined as the ratio of vortex radius to the deformation radius $R_{d}=\sqrt{g H} / f(H$ is the water depth). The strength of the beta-plane, $\beta$, depends on the tank geometry and is defined in Table 1. Equations (1.1) and (1.2) show that for small $B u$, free-surface deformation has a negligible effect on vorticity production, whereas for $B u \gg 1$, surface deformation is important. Except for the experiments of Stegner \& Zeitling (1998) (see table 1), most experiments are conducted over the parameter space $B u \ll 1$ and $R o<O(1)$.

In this paper, the dynamics of vortices characterized by $B u \ll 1,0.1<R o<1$, and $\delta \gg 1$ (see table 1) are reported. The internal structure of the monopolar vortices is varied systematically, and quantitative information about their evolution is acquired. Experimental observations on the various vortex wakes for the different initial velocity profiles are discussed in $\S 3.2$. Quantitative measurements show a relation between this initial profile and its direction of propagation ( $\$ \$ 4.1$ and 4.2 ). The influence of dominant Rossby waves on the vortex translation is discussed in $\S 4.3$. The vortex structure is discussed in comparison with a physical model incorporating the Rossby force and circulation-induced lift force in $\$ \S 5.1$ and 5.2. These results are discussed in a broader context in $\S 6$ and the conclusions are presented in $\S 7$.

\section{Experimental set-up}

The experiments were conducted in two different containers, the $13 \mathrm{~m}$ diameter Coriolis platform of LEGI, Grenoble, and a small container, whose dimensions are 
indicated in table 1. Cyclonic vortices were generated either by suction of fluid from the surface, or by stirring the fluid in a confined cylinder (see Carnevale et al. 1991). Suction typically generated vortices whose azimuthal velocity decays slowly with radius. At the level of the sink, vorticity is generated locally by stretching background vorticity while the ambient flow is essentially irrotational. The suction speed and total volume siphoned were varied, but no systematic tendency to form steeper velocity profiles for short or long suction times compared to the period of background rotation was observed.

Velocity profiles of vortices generated by stirring fluid in the cylinder were steeper than those generated by suction. Vortices created in this manner contain positive vorticity, surrounded by an outer ring of negative vorticity. Care was taken to ensure that the flow was axisymmetric before lifting the cylinder. Removing the cylinder at different stages of this decay resulted in velocity profiles with different steepness. In all cases, the velocity profile was measured shortly after their generation using particle image velocimetry as discussed below.

The vortex and the displacement of ambient fluid were visualized using the precipitation method of Honyi, Taneda \& Tatsudo (1980). This method is based on the electrolytic reaction of solder wire and results in the generation of a white powder that acts as a passive tracer. The water was made slightly salty to guide the current. The vortex was visualized by placing a solder wire either in the cylinder or near to the sink in the water. In order to generate horizontal dye lines, $0.5 \mathrm{~mm}$ diameter solder wires were spanned in the flow. As the vortex approached the wires, the voltage was turned on and the dyelines were advected, showing the displacement of the ambient fluid, thus giving qualitative information about fluid displacement and the generation of potential vorticity.

Quantitative information about the velocity field was obtained using the commercially available image processing software CIV developed by Fincham \& Spedding (1997). The fluid was seeded with $0.2 \mathrm{~mm}$ diameter particles of density $1.02 \mathrm{~g} \mathrm{~cm}^{-3}$ and was made neutrally buoyant by the addition of salt. The flow was illuminated by a horizontal laser sheet which was stable within the rotating frame of reference. Images of the experiments were captured by a digital video camera, transferred onto the hard drive of a PC by a framegrabber card and saved for post-processing.

\section{Observations and measurements}

\subsection{Dye visualizations}

In order to discern the different evolutions possible for suction vortices, a range of vortex strengths was generated. Figure 1 shows the flow evolution of an intense suction vortex $\left(\delta \approx 30, v_{\theta m} \approx 1 \mathrm{~cm} \mathrm{~s}^{-1}, R_{v m} \approx 9 \mathrm{~cm}\right)$ generated at the Coriolis platform on a beta-plane of dimensions $3.0 \times 4.5 \mathrm{~m}^{2}$ with the shallow (north) side close to the axes of rotation. To visualize how ambient fluid was advected by the vortex, a dye line was generated from east to west about $50 \mathrm{~cm}$ north of the initial position of the vortex. To the east (west) of the vortex, fluid is advected to the north (south), thus inducing positive (negative) vorticity. Initially, the dye remained compact in the vortex (figure $1 a, b$ ), suggesting that vorticity generated by the displacement of ambient fluid is entrained around the intense core-vortex. The velocity profile is steepened by negative vorticity continuously being wrapping around the vortex, which eventually causes the vortex to become elliptical by a mode two instability (figure $1 c, d$ ). The 
(a)

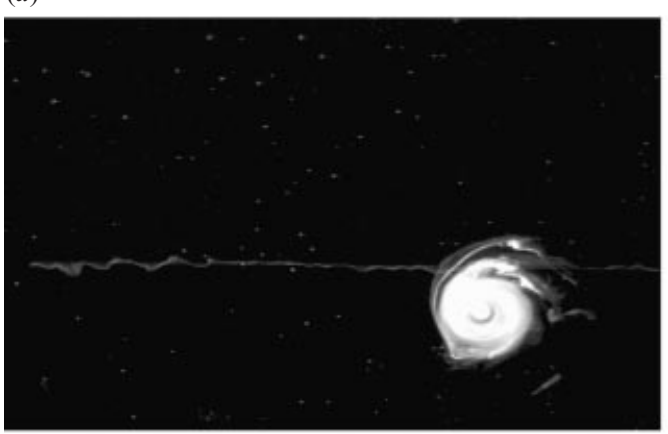

(c)

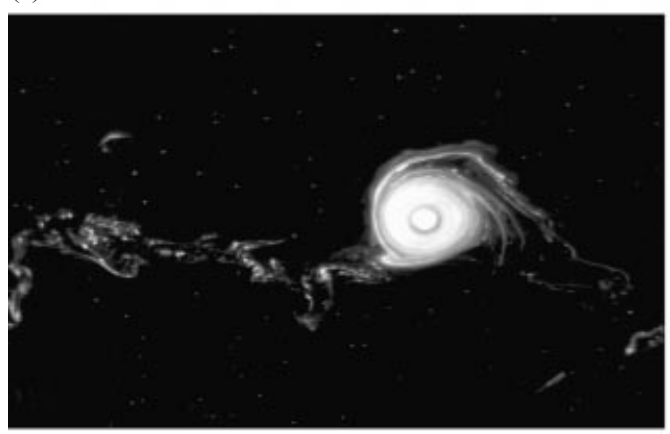

(b)

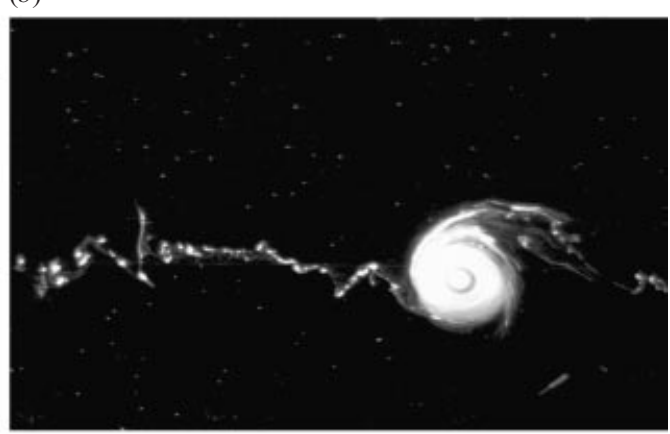

(d)

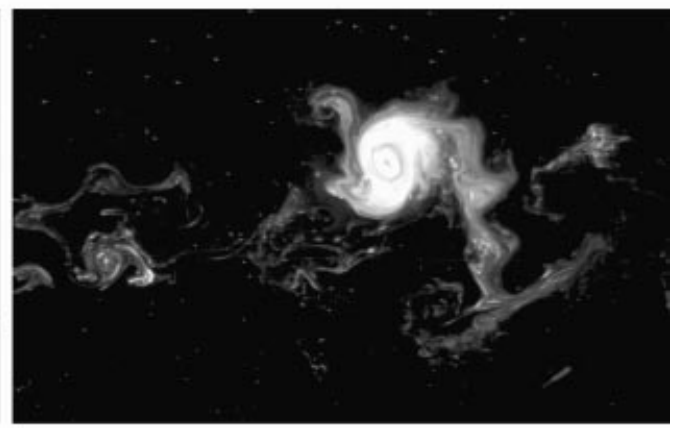

FIGURE 1. Sequence of images showing the evolution of a non-isolated (sink) vortex at $(a) 49 \mathrm{~s},(b)$ $137 \mathrm{~s},(c) 313 \mathrm{~s},(d) 739 \mathrm{~s}$ after the vortex has been generated. The rotation rate is $f=0.25 \mathrm{~s}^{-1}$, and $\beta=0.041 \mathrm{~m}^{-1} \mathrm{~s}^{-1}$. The field of view is $1.91 \times 1.38 \mathrm{~m}^{2}$ with the shallow part of the tank at the top of the figures.

herringbone-shaped wake observed is typical for isolated vortices (e.g. see Carnevale et al. 1991) and contains dyed fluid that was initially confined in the vortex.

Figure 2 shows the typical time evolution of a vortex generated by the stirring method. Owing to the steepness of the velocity profile these vortices often become barotropically unstable. The vortex core first becomes elliptical and then eventually evolves into a tripolar vortex (see Carton, Flierl \& Polvani 1989; van Heijst, Kloosterziel \& Williams 1991) as seen in figures 2(a) to 2(c). Owing to the northward displacement of the vortex and its rotation around the core centre (figure $2 a-c$ ), fluid is advected southward at the east and generates cyclonic vorticity which detrains anticyclonic vorticity from the parent vortex (figure 2). Eventually, this develops into an unstable shear layer. To the west of the vortex, the rotational motion equilibrates the vortex translation so that the dye line remains undisturbed over the course of the experiment (figure $2 c-e$ ), in contrast to sink-vortices, as shown in figure 1 . The eccentricity of the vortex changes from circular to elliptical owing to a mode two instability and finally back to circular. The secondary and tertiary vortices generated by displacing the ambient fluid form a dipole which moves to the west (figure $2 f$ ). This process is repeated until the negative vorticity that was initially around the cyclonic core is detrained and an elliptical core vortex remains that starts to move to the northwest.

\subsection{Quantitative observations of the vortex structure}

Typical vorticity contour plots of a vortex generated by the sink method are shown in figure 3 . The initial vortex is characterized by a core of intense positive vorticity. The 
(a)

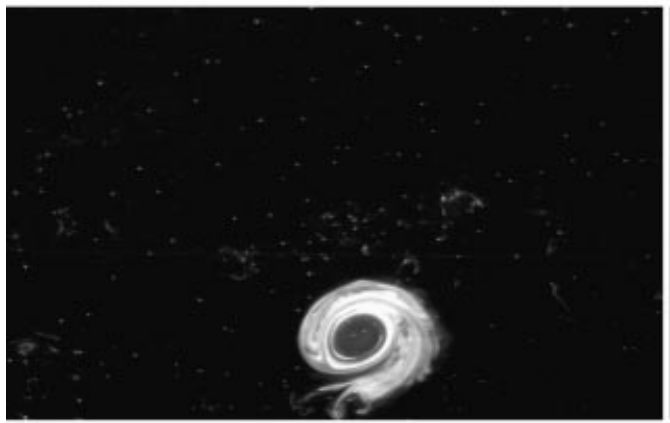

(c)

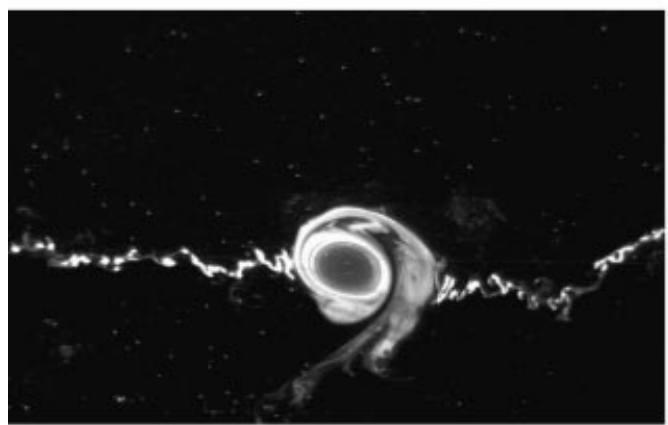

(e)

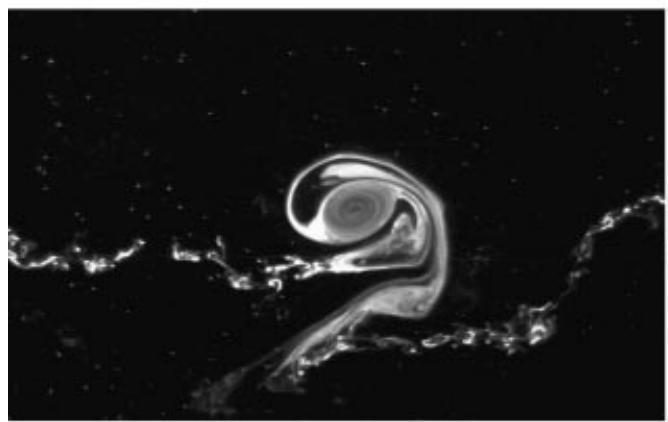

(b)

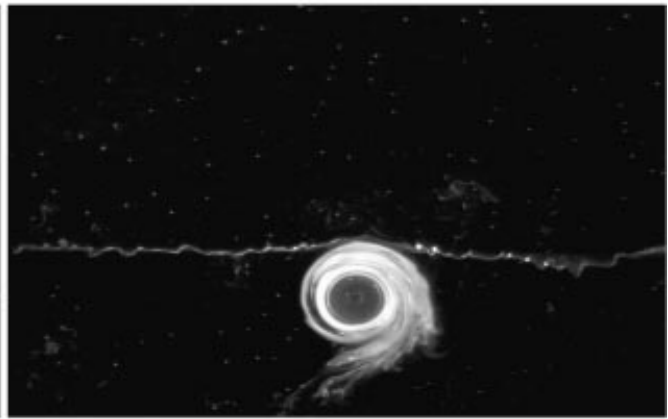

$(d)$

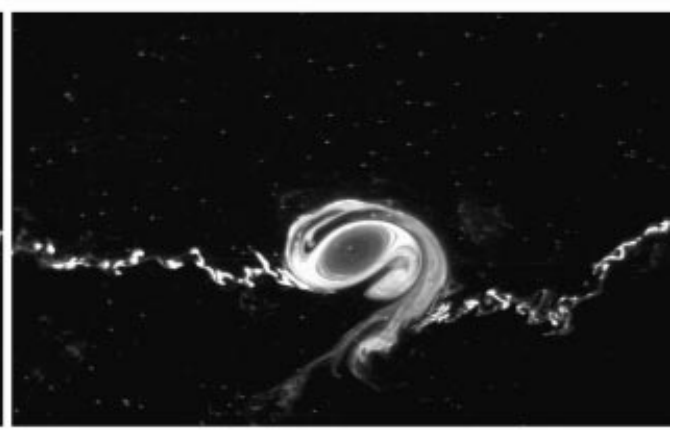

$(f)$

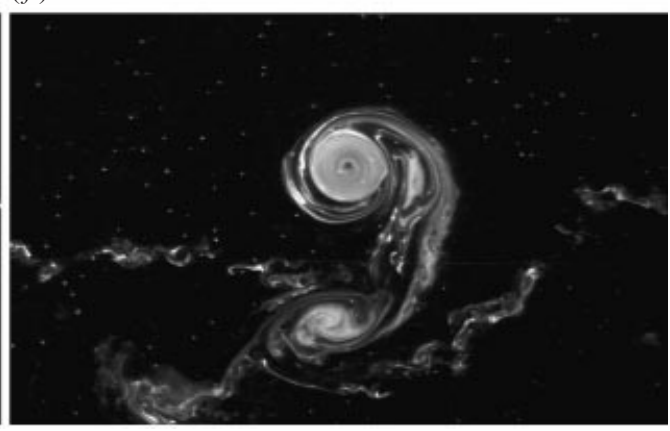

FIGURE 2. Sequence of images showing the evolution of an isolated vortex at $(a) 75 \mathrm{~s},(b) 125 \mathrm{~s},(c)$ $187 \mathrm{~s},(d) 212 \mathrm{~s},(e) 300 \mathrm{~s}$ and $(f) 488 \mathrm{~s}$ after its generation. The rotation rate is $f=0.25 \mathrm{~s}^{-1}$, and $\beta=0.0406 \mathrm{~m}^{-1} \mathrm{~s}^{-1}$. The field of view is $1.91 \times 1.38 \mathrm{~m}^{2}$.

displacement of fluid in the ambient owing to the northwest-moving vortex leads to the generation of negative vorticity which organizes into filaments that are wrapped around the core vortex, as shown in figures $3(b)$ to $3(d)$. The filaments are a typical feature of intense sink vortices and cannot be seen clearly in the dye experiments. They extend over a large region far from the initial vortex and are created by the differential rotation of iso-potential vorticity contours (Reznik 1992).

From sequences of kinetic energy density plots (not shown here), an enveloping Rossby wave was observed to propagate southwestward, perpendicular to the translation direction of the vortex. This wave is generated during the initiation of the vortex and is not immediately visible because its vorticity is initially weak compared to the vorticity in the vortex. The Rossby wave crest corresponds to the long filament 

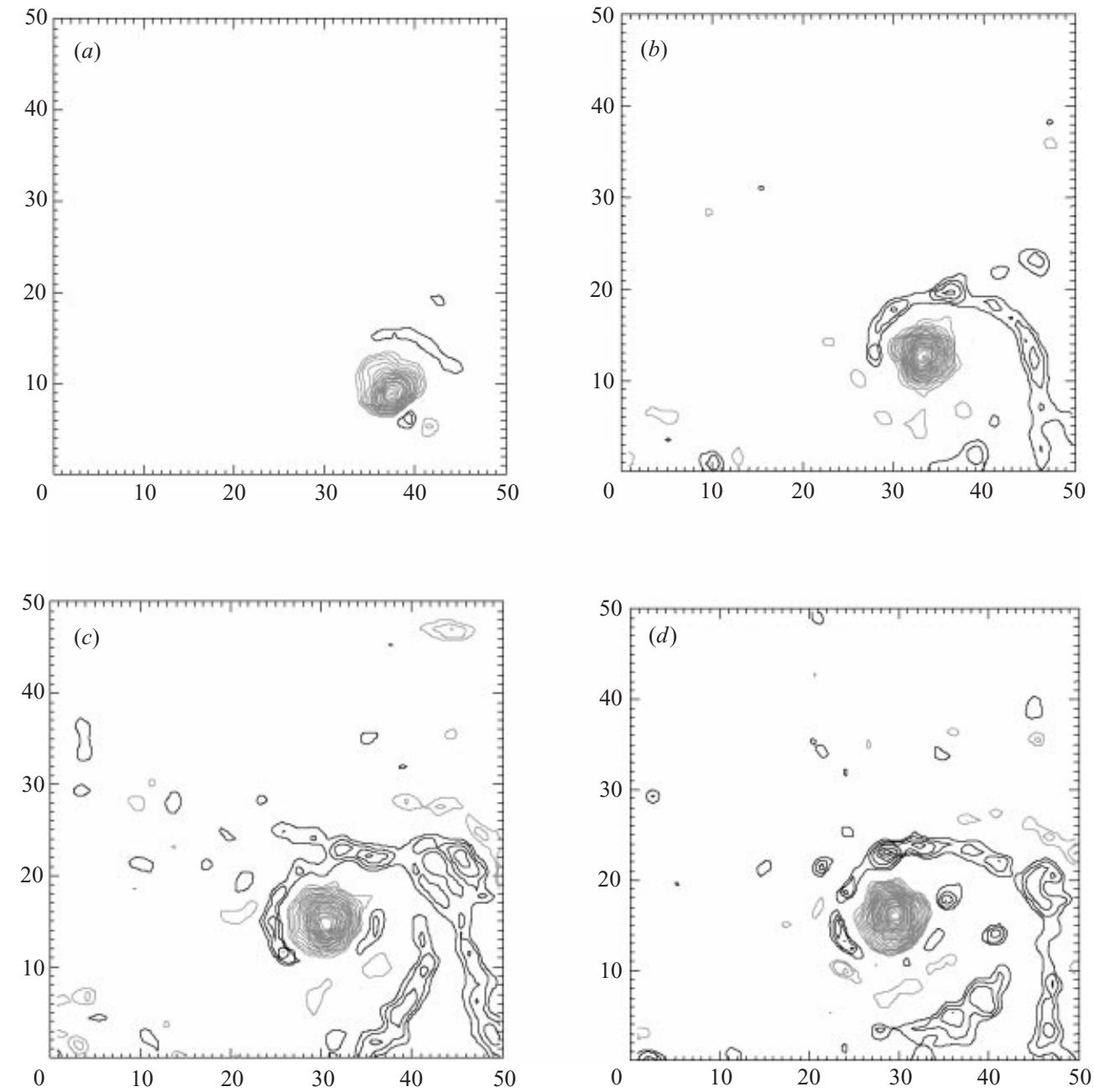

FIGURE 3. Typical vorticity contours for a sink vortex for times $(a) 1 \mathrm{~s},(b) 49.6 \mathrm{~s}(c) 86.6 \mathrm{~s}$ and (d) $111.6 \mathrm{~s}$ after the vortex has been generated. Black and grey contours represent anticyclonic (negative) and cyclonic (positive) vorticity, respectively; contour steps are 0.05 of the maximum vorticity, whereas the values around zero are omitted to suppress noise. The horizontal and vertical length scales are indicated in $\mathrm{cm}$ along the axis. The experimental observations correspond to experiment EF in table 2, where the tank rotation time is $12.5 \mathrm{~s}$ and $\beta=0.289 \mathrm{~m}^{-1} \mathrm{~s}^{-1}$.

of cyclonic vorticity generated adjacent to the vortex and envelopes the vortex as its rolling tip (see figure 3). The total wavelength is set by the diameter of the monopolar vortex, as also indicated by numerical investigations on westward moving vortices by McWilliams \& Flierl (1979). Eventually, the vortex is enclosed by a ring of anticyclonic vorticity, leading in some cases to an isolated vortex and a mode two instability (see figure $1 d$ ), but often this vorticity was too weak and the vortex remained stable.

Figure 4 shows the time evolution of the streamfunction which corresponds to the vorticity field in figure 3 . The streamfunction was corrected for the vortex translation velocity, so that the contours represent the motion in the frame moving with the vortex centre. The translation velocity of the vortex was obtained by differentiating 
(a)

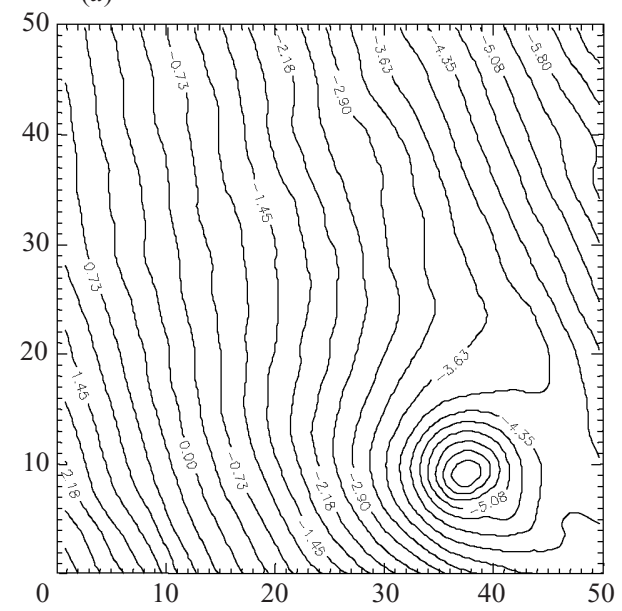

(c)

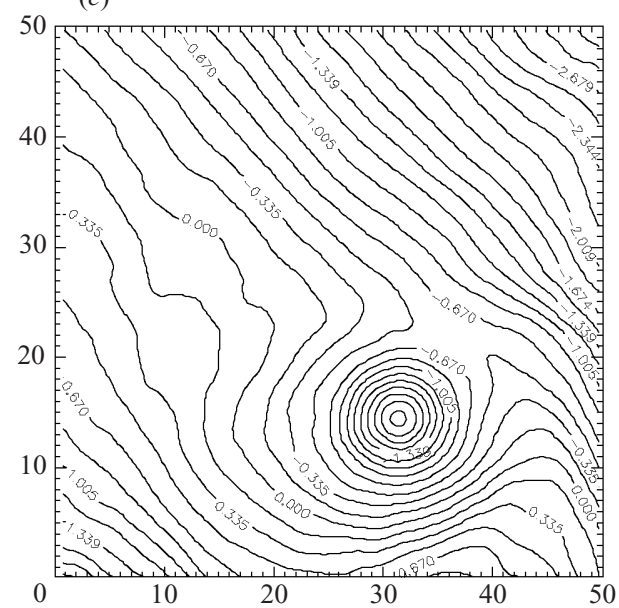

(b)

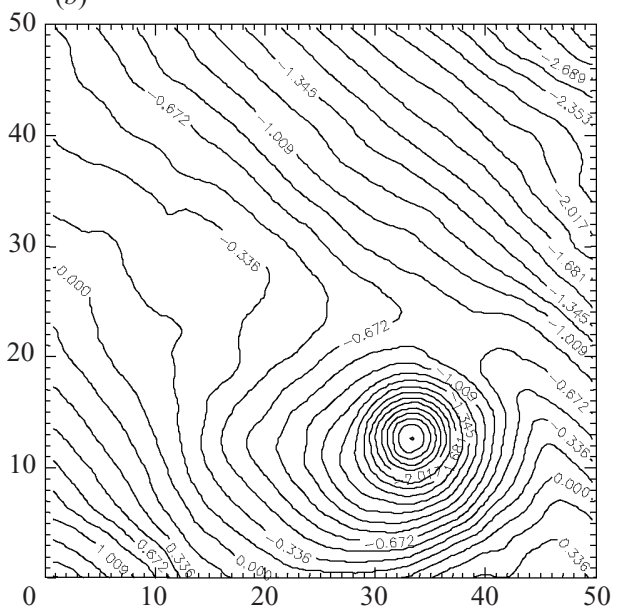

(d)

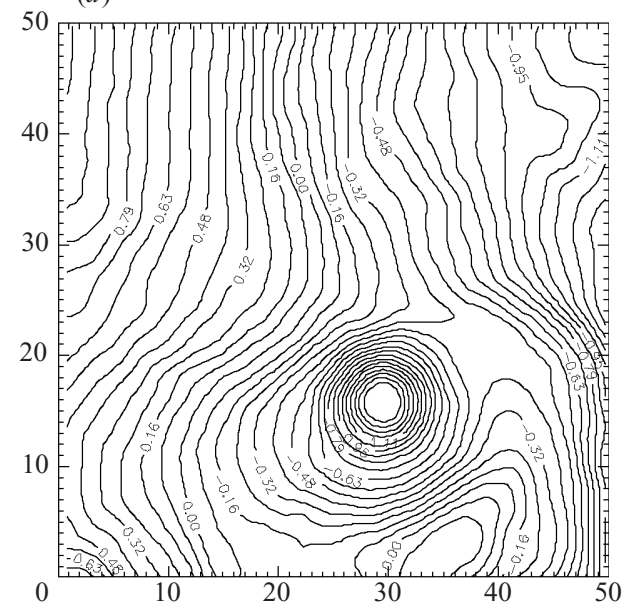

FIGURE 4. Stream function contours which are corrected for the translation velocity of the vortex and correspond to the vorticity fields in figure 3 .

the displacement of the vortex centre with respect to time, and this is discussed in more detail in $\S 3.3$. The plot indicates that the core vortex is isolated and approximately circular (figure $4 a-c$ ). The stagnation point gives information about the location and relative strength of the advected vorticity and is located approximately at the same position relative to the vortex. This suggests that the vortex moves approximately in a straight line. The convergence of the streamline contours at the east of the vortex corresponds to a region of high shear (figure $4 d$ ). At large time, the direction of motion of the vortex appears to change (as indicated in figure $4 d$ ), in accordance with the observations of Carnevale et al. (1991), and this was attributed to the reflection of Rossby waves from the tank walls.

Figure 5 shows the time evolution of the vorticity contours of an initially isolated vortex, and the corresponding streamfunction plots are shown in figure 6 . The core of the vortex is surrounded by a ring of anticyclonic vorticity. Shortly after generation, the vortex core experiences a slight westward shift, as can be inferred from figure $5(b)$. 

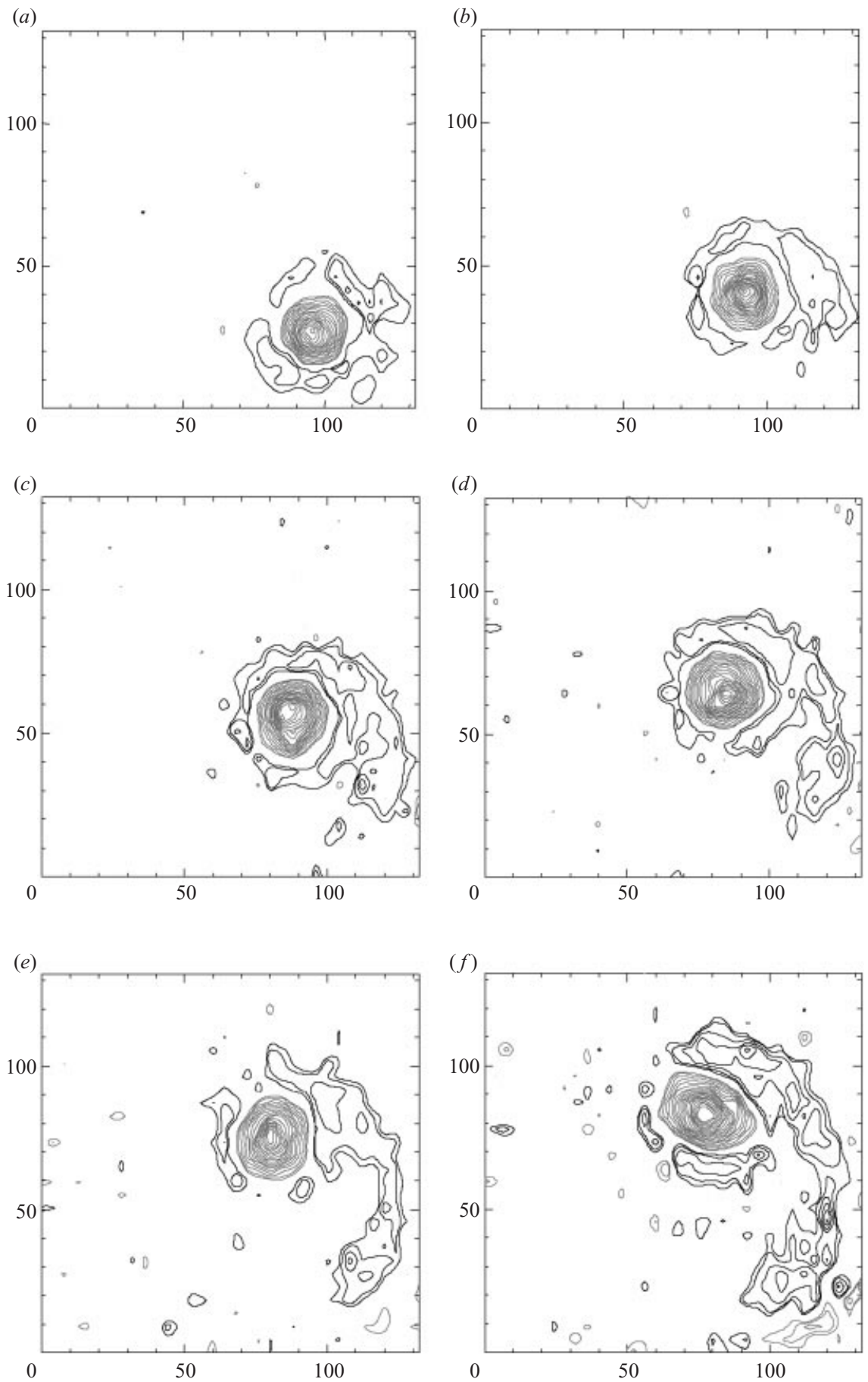

FiguRE 5. Contour plots of the vorticity for an isolated vortex generated by the stirring method for (a) $60 \mathrm{~s}$; (b) $87.5 \mathrm{~s}$; (c) $125 \mathrm{~s}$; (d) $150 \mathrm{~s}$; (e) $175 \mathrm{~s}$; and $(f) 200 \mathrm{~s}$ after forcing. The internal diameter of the cylinder used to generate the vortex is $50 \mathrm{~cm}$. Contour steps are chosen $0.05 \omega_{\max }$ and $0.07 \omega_{\max }$ around zero to suppress the noise; black and grey contours represent anticyclonic (negative) and cyclonic (positive) vorticity, respectively. Vertical and horizontal length scales are indicated in $\mathrm{cm}$ along the axis. Parameters are $f=0.25 \mathrm{~s}$ and $\beta=0.0406 \mathrm{~m}^{-1} \mathrm{~s}^{-1}$. Experiment AH in table 2 . 
Reorganization of anticyclonic vorticity leads to an asymmetric dipolar-like vortex which is clearly visible in the vorticity contours (figure $5 b-d$ ) and the corresponding streamfunction contours (figure $6 b-d$ ). In contrast to most asymmetric dipolar vortex structures, which move along a curved path, the vortex structure in figure 5 moves along an approximately straight line to the north, suggesting a quasi-monopolar structure (Stern \& Radko 1998) or a Chaplygin dipolar structure (see Meleshko \& van Heijst 1994). As can be inferred from figure $6(d-f)$, cyclonic vorticity generated at the east detrains part of the ring vorticity into the long wake. The cyclonic vorticity is weak and not resolved by the contours in figure 5, but since the cyclonic vortex is large, the circulation is not negligible (see comments relating to figure 2 ). The vortex eventually evolves into a tripolar-like structure, as shown in figures $5(f)$ and $2(c)$, with an elliptical core and two patches of anticyclonic vorticity. The patch of anticyclonic vorticity south of the vortex has half the value in vorticity of the patch north of the vortex and is therefore of minor influence on the vortex trajectory which continues to move to the north.

\section{Vortex trajectory and translation speed}

\subsection{Characterization of the initial vortex}

The vortex centre, defined by the vorticity maximum, was determined by taking a spline approximation to the vorticity field around the vorticity maximum. Crosssections of velocity and vorticity were taken through the vorticity maximum along lines parallel to the east-west direction. Figures $7(a)$ and $7(b)$ show time sequences of the cross-sectional velocity profile of a vortex generated by the stirring and sink method, respectively. The velocity profiles are shifted along the $x$-axis, so that $x=0$ corresponds to the vorticity maximum. The velocity is normalized by the minimum velocity $\left(\left|v_{\theta \min }\right|\right)$ but the horizontal axis, representing the radial distance from the vortex centre, is kept in dimensional form to indicate the size of the vortex. The velocity profiles for the vortex generated by stirring (figure $7 a$ ) show an initially symmetric profile and subsequently an increase of maximium velocity, followed by a decrease at the eastside. This is due to the presence of an anticyclonic vortex (figure $6 b, c$ ) that causes a dipolar motion. The corresponding profiles for the sink vortex become asymmetric due to the generation of Rossby waves in the far field. This leads to a much larger northward velocity, known as a beta-gyre (Sutyrin \& Flierl 1994). Figures 7(a) and 7(b) indicate that the change in the diameter of the vortex core, represented by the distance between the maximum and minimum values of velocity, is negligible over the time scale of the experiment.

In order to characterize the vortex velocity profile after its generation, the experimental velocity profiles were fitted by a similarity solution of the radial diffusion equation on the $f$-plane,

$$
\hat{v}_{\theta}(\hat{r})=\frac{1}{\hat{r}} \exp \left(-\frac{1}{4} \hat{r}^{2}\right) W_{\frac{1}{2}(\alpha-1), \frac{1}{2}}\left(\frac{1}{2} \hat{r}^{2}\right)
$$

where $\hat{r}=r / R_{v m}, \hat{v}_{\theta}=v_{\theta} / v_{\theta m}$ and $W$ is the Whittaker function (Gradshteyn \& Ryzhik 1980 p. 157). The velocity profile of an isolated vortex $(\alpha=3)$ is given by

$$
\hat{v}_{\theta}=\hat{r} \exp \left(-\frac{1}{2} \hat{r}^{2}\right)
$$

whereas for $\alpha=1$ we obtain a velocity profile for a non-isolated (Gaussian) vortex

$$
\hat{v}_{\theta}=\frac{1}{\hat{r}}\left(1-\exp \left(-\frac{1}{2} \hat{r}^{2}\right)\right)
$$



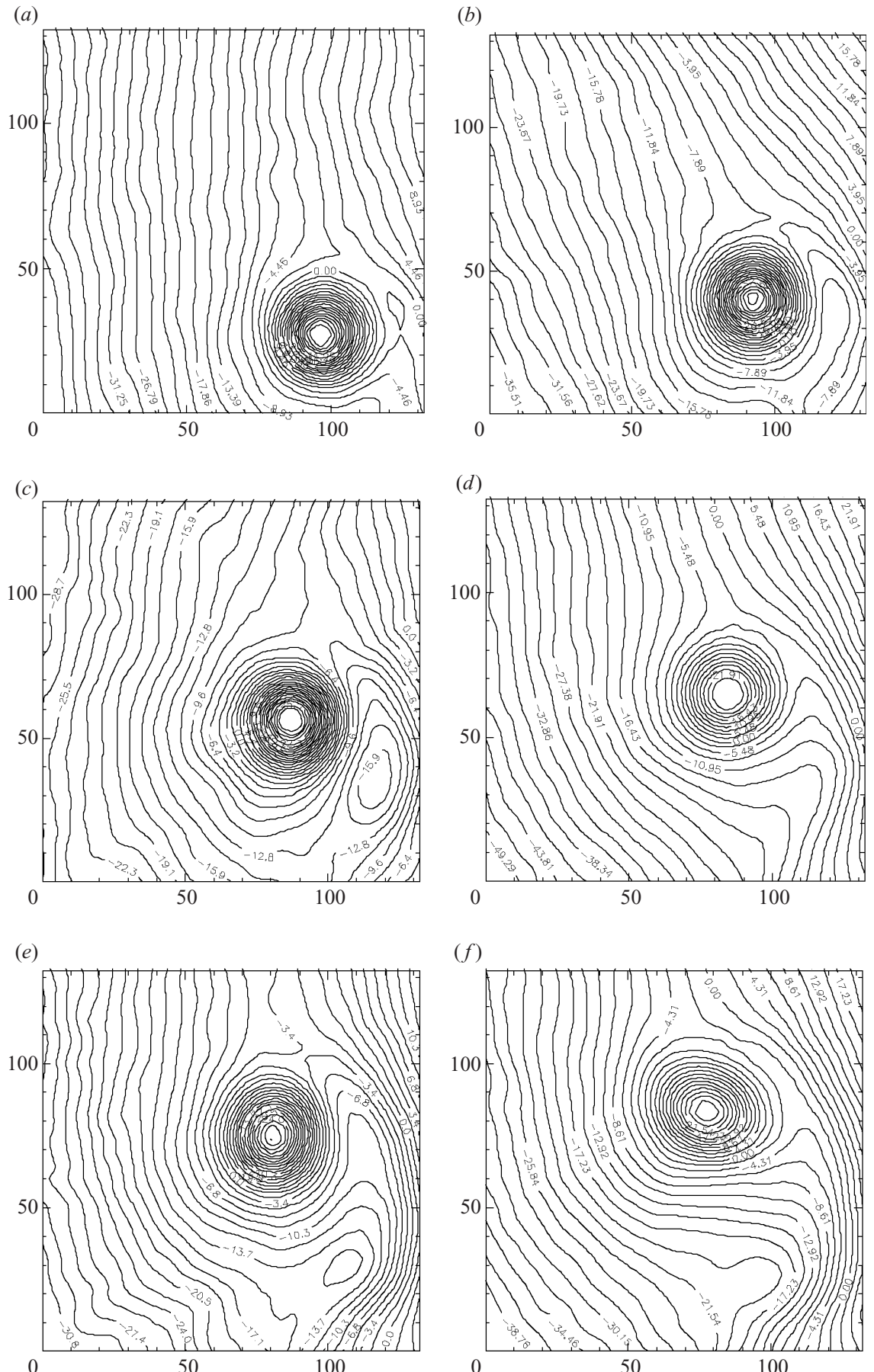

FIGURE 6. Streamfunction contours corresponding to the vorticity field shown in figure 5, after corection for the vortex translation. 

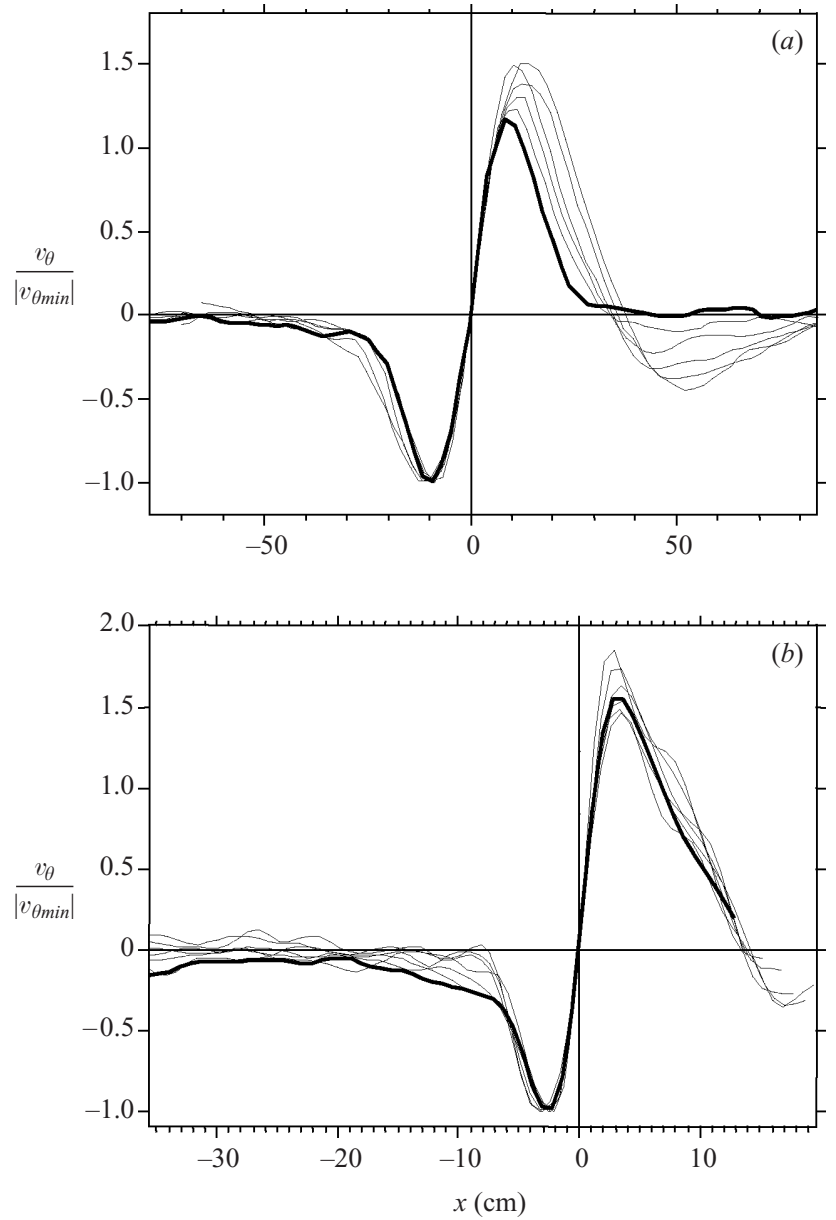

FIGURE 7. Typical measurements of the azimuthal velocity along an east-west cross-section through the vorticity maximum for $(a)$ an isolated vortex $(b)$ a quasi-non-isolated. The bold line indicates the initial profile. The time difference between each pair of profiles is one rotation period which corresponds to $50 \mathrm{~s}$ (at Coriolis platform) and $12.5 \mathrm{~s}$ (small turntable) for $(a)$ and $(b)$ respectively.

Equation (4.1) represents a continuous range of velocity profiles with the steepness increasing monotonically with $\alpha$. Although this family of similarity solutions is strictly valid for an $f$-plane, on a time period smaller than $1 /\left(\beta R_{v m}\right)$, over which the beta effect is expected to be relatively small, the initial vortex structure is described to leading order by (4.1). The initial velocity profile was compared with similarity solutions over the range $r / R_{v m}<3.0$, and $\alpha$ was determined from the curve of best-fit, of which two typical examples are shown in figure 8 . From these fits, the vortex was characterized by $R_{v m}, v_{m}$ and $\alpha$. Vortices generated by the stirring method are characterized by $1.4<\alpha \leqslant 3$, whereas vortices generated by suction are characterized by $\alpha$ close to 1 . Table 2 summarizes the measured properties of the monopolar vortices characterized in the experimental study.

\subsection{Trajectory of the vortex centre}

Figure $9(a)$ shows the vortex trajectory for four different values of $\alpha$. Vortices generated by a sink (characterized by $\alpha<1.4$ ) move along a straight line to the northwest, whereas vortices generated by stirring $(1.4<\alpha<3)$ show a tendency to move more 


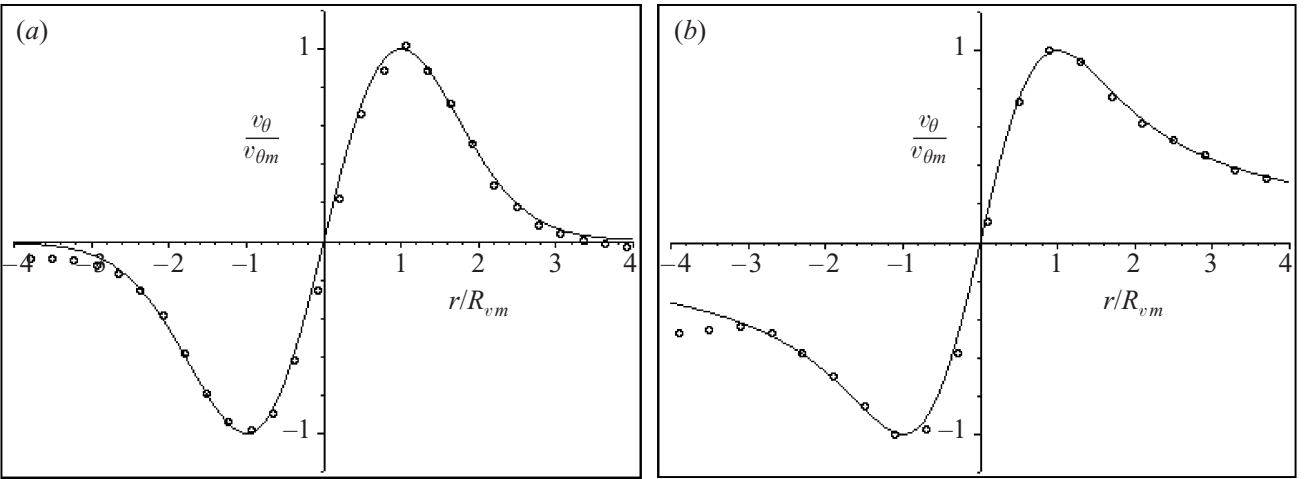

FIGURE 8. Typical cross-sectional velocity profiles along an isoline of potential vorticity fitted with the profile given by equation (4.1) for $(a) \alpha=2.9$ and $(b) \alpha=1.1$.

\begin{tabular}{llllcccccr}
\hline Exp. & $\alpha$ & $\mathscr{R}^{2}$ & $\theta \pm 5$ & $R_{v m}(\mathrm{~cm})$ & $v_{\theta m}\left(\mathrm{~cm} \mathrm{~s}^{-1}\right)$ & $\beta\left(\mathrm{m}^{-1} \mathrm{~s}^{-1}\right)$ & $U\left(\mathrm{~cm} \mathrm{~s}^{-1}\right)$ & $R o=\frac{v_{\theta m}}{f R_{v m}}$ & $\delta$ \\
AA & 3.0 & 0.99 & 90 & 13.8 & 2.1 & 0.0406 & 0.21 & 0.61 & 27 \\
AB & 3.0 & 0.99 & 85 & 12.9 & 2.0 & 0.0406 & 0.20 & 0.62 & 30 \\
AC & 2.3 & 0.99 & 72 & 12.8 & 1.8 & 0.0406 & 0.22 & 0.56 & 27 \\
AE & 3.0 & 0.97 & 90 & 12.0 & 1.9 & 0.0406 & 0.18 & 0.63 & 32 \\
AH & 2.7 & 0.997 & 77 & 11.2 & 3.2 & 0.0406 & 0.32 & 1.14 & 63 \\
AI & 1.4 & 0.985 & 51 & 9.4 & 0.6 & 0.0406 & 0.16 & 0.26 & 17 \\
AJ & 2.6 & 0.996 & 75 & 9.7 & 1.6 & 0.0406 & 0.20 & 0.66 & 42 \\
AL & 3.0 & 0.999 & 90 & 9.1 & 1.8 & 0.0406 & 0.21 & 0.79 & 54 \\
AO & 3.0 & 0.982 & 90 & 8.0 & 0.3 & 0.0406 & 0.12 & 0.15 & 12 \\
AP & 1.6 & 0.991 & 62 & 10.0 & 1.0 & 0.0406 & 0.19 & 0.40 & 25 \\
AQ & 3.0 & 0.97 & 78 & 11.6 & 3.5 & 0.0406 & 0.41 & 1.21 & 64 \\
EB & 0.9 & 0.9995 & 34 & 3.9 & 1.05 & 0.289 & 0.27 & 0.27 & 24 \\
EC & 1.2 & 0.996 & 40 & 3.5 & 0.94 & 0.289 & 0.22 & 0.27 & 27 \\
ED & 1.1 & 0.998 & 48 & 2.5 & 0.63 & 0.289 & 0.11 & 0.25 & 35 \\
EE & 1.2 & 0.99 & 51 & 3.3 & 0.45 & 0.289 & 0.13 & 0.14 & 14 \\
EF & 1.1 & 0.997 & 40 & 3.2 & 0.47 & 0.289 & 0.13 & 0.15 & 16 \\
EG & 1.1 & 0.998 & 45.7 & 2.5 & 1.5 & 0.289 & 0.28 & 0.6 & 83 \\
EH & 1.2 & 0.998 & 36.5 & 2.6 & 2.1 & 0.289 & 0.32 & 0.81 & 107 \\
FA & 1.4 & 0.996 & 57 & 3.5 & 0.8 & 0.289 & 0.23 & 0.23 & 23 \\
FB & 1.2 & 0.998 & 49 & 2.5 & 1.25 & 0.289 & 0.24 & 0.5 & 69 \\
FC & 1.1 & 0.997 & 48 & 2.9 & 1.12 & 0.289 & 0.22 & 0.39 & 46 \\
FD & 1.4 & 0.995 & 50 & 3 & 0.9 & 0.289 & 0.21 & 0.3 & 35 \\
FE & 1.5 & 0.996 & 49 & 2.5 & 1.3 & 0.289 & 0.23 & 0.52 & 72 \\
FF & 0.9 & 0.992 & 37 & 4.7 & 0.5 & 0.289 & 0.19 & 0.11 & 8 \\
GA & 0.8 & 0.998 & 40 & 4.7 & 1.15 & 0.289 & 0.32 & 0.24 & 18 \\
GB & 1.3 & 0.993 & 52.8 & 2.5 & 1.2 & 0.289 & 0.18 & 0.48 & 66 \\
GC & 1.0 & 0.997 & 33.2 & 5 & 0.95 & 0.289 & 0.35 & 0.19 & 13
\end{tabular}

TABLE 2. Initial vortex parameters measured after their generation by means of PIV. The experiments with $\beta=0.0406 \mathrm{~m}^{-1} \mathrm{~s}^{-1}$ - with initial $\mathrm{A}$-represent experiments performed on the $13 \mathrm{~m}$ diameter Coriolis turntable, other experiments - with initials $\mathrm{E}, \mathrm{F}$ and $\mathrm{G}$-are performed on the $1 \mathrm{~m}^{2}$ turntable. $\mathscr{R}^{2}$ is the correlation value of the fit of similarity solution for the velocity profile to the experimental measurements, of which typical examples are shown in figure 8 , and $\theta$ represents the angle between the west and northward direction of propagation. The translation speed $U$ is obtained by differentiating the position of relative vorticity maximum with respect to time. The speed shown in the table is determined by averaging over ten measurements and showed a variation of typically $50 \%$. Experimental series E, F and G and experiments AI and AP are generated by the sink method, in other experiments the stirring method is used. 

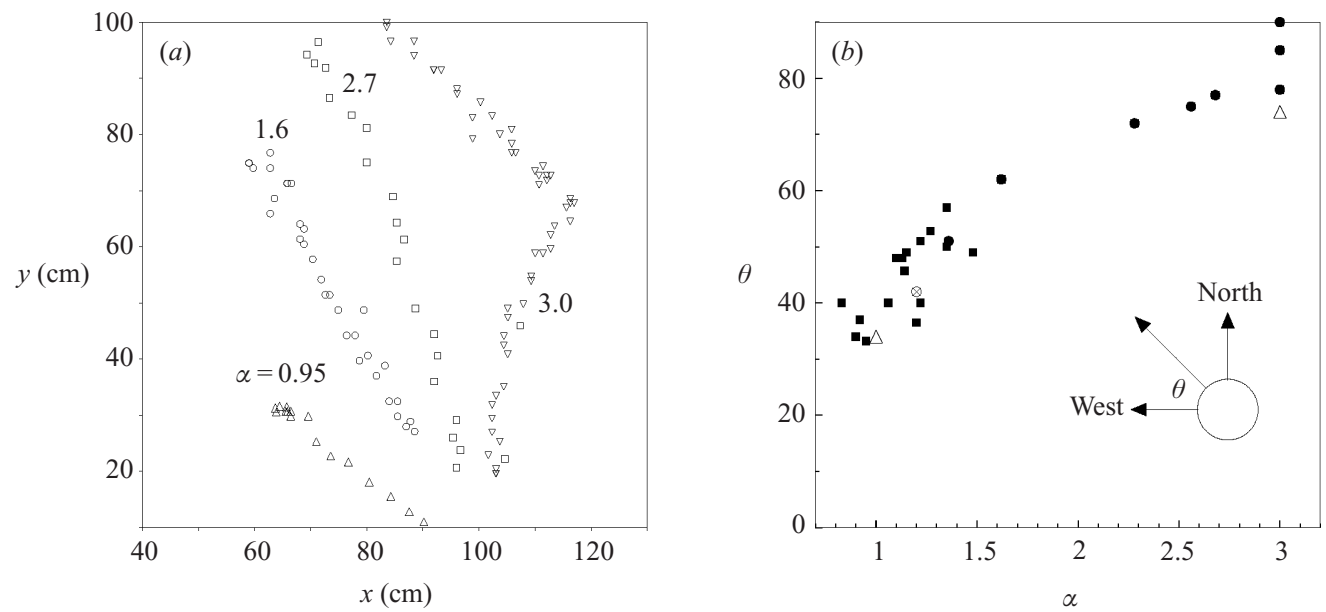

FIGURE 9. (a) Trajectory of vortex centre for various values of $\alpha=0.95,1.6,2.7$ and 3.0. The time step between the data points is $12.5 \mathrm{~s}$. $(b)$ The variation of the angle the trajectory makes to the west, $\theta$, with $\alpha$ (measured from fits shown in figure 8 ). The circles show the experiments conducted at the Coriolis platform, the squares represent the experiments on the square table and concern all sink vortices. $(\otimes)$ the value obtained for a stirred vortex that has lost its anticyclonic vorticity, represented by $\alpha=3.0$ in $(a)$. $\triangle$, numerical results of Carnevale et al. (1991) for $\alpha=1.0$ and 3.0.

northward. The large radial gradient of the azimuthal velocity of vortices characterized by $\alpha \approx 3$ means that they become unstable and develop into a tripolar vortex (denoted by $\nabla$ in figure $9 a$ ). When the ring of anticyclonic vorticity is eventually detrained into the wake, the vortex propagates to the northwest in the same direction as vortices characterized by $\alpha \approx 1$ (see figure $9 a$ ). Measurements of the velocity profile at this stage of the flow evolution reveal a comparable $\alpha$-value to sink vortices.

The direction of motion of the vortex centre was averaged over two background rotation periods. Figure $9(b)$ shows the variation of the angle the trajectory makes with the west, and indicates that $\theta$ increases with $\alpha$. For $\alpha=3$, the vortex is often barotropically unstable, as mentioned above, and either the secondary vortices of the tripole, or the anticyclonic vortices shed in the wake induce a dipolar moment on the parent vortex to the east (see figures $2(f)$ and $5(f)$ ). This leads to a propagation direction that is at variance with the tendency in the data (see figure $9 b$ ). For values of $\alpha<3$, the vortex remained stable. Results inferred from the numerical simulations of Carnevale et al. (1991) for the initial direction of motion of monopolar vortices, characterized by $\alpha=1$ and 3, are plotted in figure $9(b)$, showing a reasonable agreement with the experimental results. It is important to note that the dimensionless parameter $\delta=v_{\theta m} / \beta R_{v m}^{2}$ varies considerably over the experiments (see table 2 and figure $10 a$ ) and is not correlated to the direction of motion. Since the strength of the beta-plane is essential for vortex motion, it may appear counterintuitive that the initial direction of motion does not depend on $\beta$. However, the translational speed of the vortex depends on $\beta$ and is zero in the absence of a beta-plane.

\subsection{The vortex translation speed}

The translational velocity of the vortex was measured from the displacement of the maximum of vorticity and was averaged over four tank rotation periods after the vortices were generated. Figure 10(a) shows the variation of the translation speed of the vortex with the maximum azimuthal velocity, both scaled by $\beta R_{v m}^{2}$; the vortices 

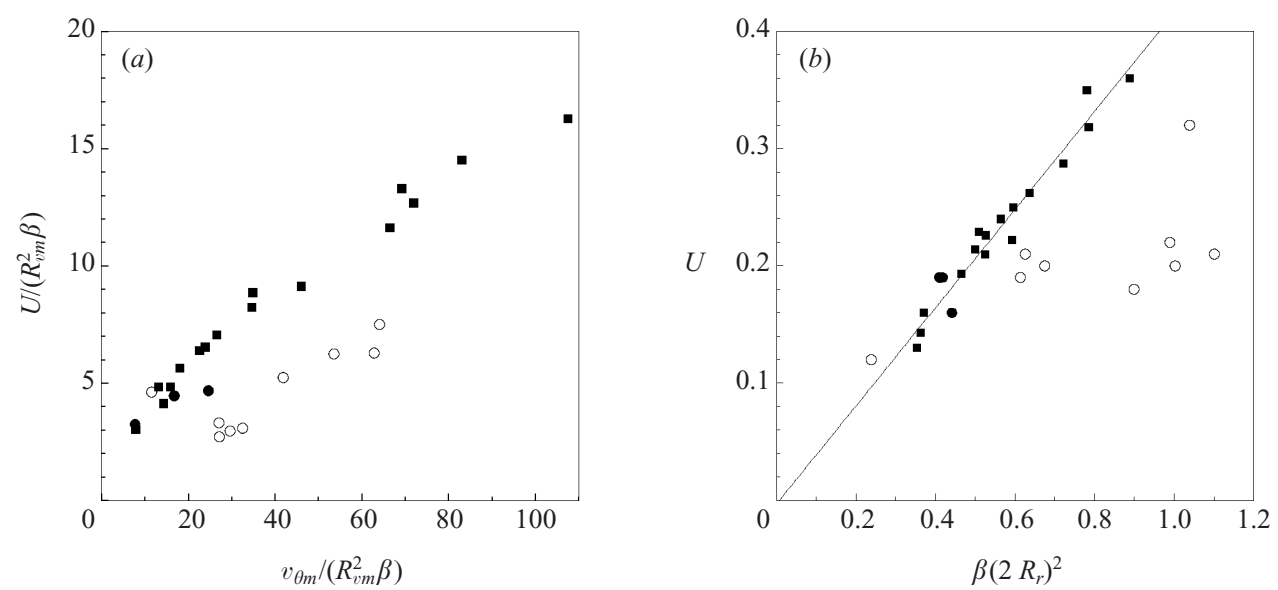

FIGURE 10. (a) Variation of the translational speed of the vortex $U$ with the maximum azimuthal velocity $v_{\theta m}$, where both velocities have been scaled with $\beta R_{v m}^{2}$. In both plots, the circular dots represent vortices generated on the Coriolis platform and the square dots represent vortices generated on the $1 \mathrm{~m}^{2}$ tank; closed symbols represent vortices with $\alpha<1.4$ and open symbols represent vortices with $\alpha>1.4$. The propagation speed of the sink vortices was fitted with a correlation $U / \beta R_{v m}^{2}=0.8\left(v_{\theta m} / \beta R_{v m}^{2}\right)^{0.64}$ (with correlation coefficient $\left.=0.96\right)$. $(b)$ Shows a comparison between the predicted speed of the Rossby waves $\sim \beta\left(2 R_{r}\right)^{2}$ and the translation speed of the vortex which is discussed in the text.

generated by the sink and stirring methods are distinguished by closed and open symbols, respectively. Vortices generated by stirring are characterized by $\alpha>1.4$ and move slower than sink vortices, as also noted by Carnevale et al. (1991). The difference in the propagation speed principally arises from Rossby waves which interact with the vortex. Rossby waves may be generated by the translational motion or the azimuthal flow of the vortex. However, since the vortex moves faster than long Rossby waves (see figure 10), the waves are predominantly generated by the azimuthal flow generated by the vortices. The initial velocity profile of sink vortices (characterized by $\alpha<1.4$ ) decays slowly with distance from the vortex centre and therefore generates a perturbation to the potential vorticity field over a large area, leading to relatively fast Rossby waves, which were observed to be in phase with the vortex motion. In order that the azimuthal velocity field is in phase with the Rossby waves, the azimuthal speed must match the Rossby wave phase speed at a distance $R_{r}$ from the vortex core, so that

$$
v_{\theta}\left(R_{r}\right)=\beta\left(2 R_{r}\right)^{2},
$$

must be satisfied. Combining (4.1) with (4.4) enables the evaluation of the wave length of the Rossby waves, $2 R_{r}$, and implicitly their phase speed $c_{p h}=\beta\left(2 R_{r}\right)^{2}$ for each value of $\alpha$ and $v_{\theta m}$. Comparisons between the phase speed of the Rossby waves, based on a wavelength $2 R_{r}$, with the translation velocity of the vortex $U$ are shown in figure $10(b)$ and show good agreement with $U=0.4 \beta\left(2 R_{r}\right)^{2}$ (correlation $\mathscr{R}^{2}=0.96$ ) for vortices with $\alpha<1$.4. Experimental observations discussed above strongly suggest that the vortex translates in the direction of energy propagation (i.e. direction of the group waves), and not in the direction of the phase speed, which may explain why the coefficient is close to $\frac{1}{2}$. This correlation underlines once more the dominant effect of the Rossby waves on the dynamics in contrast to compact vortices with $\alpha>1.4$, which are characterized by an azimuthal velocity which decays rapidly with distance, so that energy associated with the Rossby waves is small. 


\section{Mechanistic model of the monopolar vortex}

\subsection{The model}

A number of approaches have been applied to describe the dynamics of monopolar vortices on the beta-plane, including formal asymptotic expansions of the vorticity equation in $\beta R_{v m}^{2} / v_{\theta m}$ (Reznik \& Dewar 1994) or dimensionless time (Adem 1956). Rossby (1948) originally adopted a mechanistic approach to explain the phenomenon of monopolar vortex motion on the beta-plane by treating the vortex as a circular patch of vorticity subject to the Coriolis force, which after integrating over the vortex area leads to a northward (Rossby) force

$$
F_{R}=\rho \beta \int_{0}^{R} \pi r^{2} v_{\theta} \mathrm{d} r
$$

where $R$ is the vortex radius and $\rho$ is the density of the ambient fluid (see $\S 1$ ).

In this paper, the Rossby model is generalized by the inclusion of additional forces. When the circulation around the vortex $\Gamma\left(=2 \pi R v_{\theta}(R)\right)$ is zero, the only force acting on the vortex is the Rossby force which is directed towards the north. However, when $v_{\theta}(R)>0$, the circular patch has a positive circulation and coupled with the translation is subject to the lift force. This force arises from a velocity difference (and pressure drop) between east and west of the vortex, resulting from the combined effect of the northward motion of the patch and the circulation around it. The lift force may be interpreted as arising from equal contributions from the momentum flux far from the cylinder and the pressure force (see e.g. Batchelor 1967, p. 407). The lift force was not included in the seminal work of Rossby.

We implicitly assume that the vorticity generated inside the vortex is small compared to the vorticity present in the vortex patch (i.e. $\delta \gg 1$ ), so that our model applies to intense vortices. As with Rossby, we assume a potential flow around the vortex. For isolated vortices this advected vorticity is small, but its importance increases for vortices with weaker decaying velocity profiles, i.e. smaller $\alpha$-values. The validity of the model with respect to $\alpha$ will be discussed later.

It is relevant to note the analytical model of Korotaev \& Fedotov (1994) on the motion of Gaussian monopolar vortices (i.e. $\alpha=1$ ). Their physical model reduces to a quasi-steady balance between the Rossby force directed northward, and, in contrast to the present model, a southward lift force generated by the circulation around a westward moving vortex.

The total force on a circular vortex arises from the Rossby force, the lift force due to circulation $\Gamma$, and the added-mass force due to the displacement of fluid by the vortex as it moves around. This flow is identical to the potential flow around a circular cylinder containing circulation and subjected to an external force. The general case of this classical flow problem is treated by Lamb (1932 p. 80). Below, we derive the integrated equations of motion from the Euler equations in the rotating frame of reference given by

$$
\frac{\mathrm{D} \boldsymbol{u}}{\mathrm{D} t}=-\nabla p-2 \Omega(y) \hat{z} \times \boldsymbol{u} .
$$

The translative motion of the vortex is in good approximation geostrophic (since the Rossby number defined by the vortex translation speed $U$ is $U / f R_{v m} \ll 1$ ) so that to leading order the pressure force is balanced by the Coriolis force on the moving vortex. The total force on a circular vortex of radius $R$, density $\rho$, obtained by integrating the pressure variation over the surface of the vortex and adding the 
Rossby force $F_{R} \hat{y}$ become

$$
\rho \pi R^{2} \frac{\mathrm{d} \boldsymbol{v}}{\mathrm{d} t}=-\int_{S} p \hat{\boldsymbol{n}} \mathrm{d} S+F_{R} \hat{\boldsymbol{y}},
$$

where $\hat{\boldsymbol{n}}$ is the unit vector normal to (and out of) the surface of the vortex and $\boldsymbol{v}=(u, v)$ is the translation velocity of the vortex. The contribution from the pressure variation over the vortex surface may be calculated by evaluating the momentum flux far from the vortex. When the outer flow is to leading order irrotational, this force is

$$
-\int_{S} p \hat{\boldsymbol{n}} \mathrm{d} S=-\rho \pi R^{2} \frac{\mathrm{d} \boldsymbol{v}}{\mathrm{d} t}+\rho \Gamma \hat{z} \times \boldsymbol{v},
$$

which correspond, respectively, to the added-mass force of a cylinder and the lift force induced by the circulation. Combining (5.3) and (5.4), we find that the dynamics of a circular vortex are to leading order described by

$$
2 \rho \pi R^{2} \frac{\mathrm{d} \boldsymbol{v}}{\mathrm{d} t}=\rho \Gamma \hat{z} \times \mathbf{v}+\boldsymbol{F}_{R} \hat{\boldsymbol{y}}
$$

Integrating (5.5) with respect to time, subject to the initial condition that the vortex starts from rest at the origin, gives

$$
u=-\frac{F_{R}}{\rho \Gamma}\left(1-\cos \left(\frac{\Gamma t}{2 \pi R^{2}}\right)\right), \quad v=\frac{F_{R}}{\rho \Gamma} \sin \left(\frac{\Gamma t}{2 \pi R^{2}}\right) .
$$

The translation speed $U$ and direction of motion $\theta$ to the west calculated from (5.6) are

$$
U=\sqrt{u^{2}+v^{2}}=2 \frac{F_{R}}{\rho \Gamma} \sin \left(\frac{\Gamma t}{4 \pi R^{2}}\right), \quad \theta=\tan ^{-1}\left(-\frac{v}{u}\right)=\frac{\pi}{2}-\frac{\Gamma t}{4 \pi R^{2}} .
$$

Substituting the expressions for the Rossby force $F_{R}$ (5.1) and circulation $\Gamma$, the translational speed and direction of motion are

$$
U=\beta \frac{\int_{0}^{R} r^{2} v_{\theta} \mathrm{d} r}{R v_{\theta}(R)} \sin \left(\frac{v_{\theta}(R) t}{2 R}\right), \quad \theta=\frac{1}{2} \pi-\frac{v_{\theta}(R) t}{2 R} .
$$

According to (5.8), the vortex speed is determined by the strength of the beta-plane, but the direction is independent of $\beta$. The trajectory of the vortex centre is

$$
x(t)=-\frac{2 \pi R^{2} F_{R}}{\rho \Gamma^{2}}\left(\frac{\Gamma t}{2 \pi R^{2}}-\sin \left(\frac{\Gamma t}{2 \pi R^{2}}\right)\right), \quad y(t)=-\frac{2 \pi R^{2} F_{R}}{\rho \Gamma^{2}}\left(1-\cos \left(\frac{\Gamma t}{2 \pi R^{2}}\right)\right) .
$$

The vortex follows a trochoid trajectory between its initial latitude and the north. According to the model above, the direction of motion changes with time because a balance between the forces acting on the vortex is not achieved.

The strength of this simple model is that it appears to capture the gross dynamics of monopolar vortices on a beta-plane: the initial direction of motion of the vortex depends on the azimuthal velocity profile through $\alpha$, which is a measure of the steepness of the velocity profile. The initial vorticity distribution within the vortex essentially controls the ratio of the Rossby force, driving the vortex north, and the lift force which drives the vortex west. This explains physically why compact vortices 

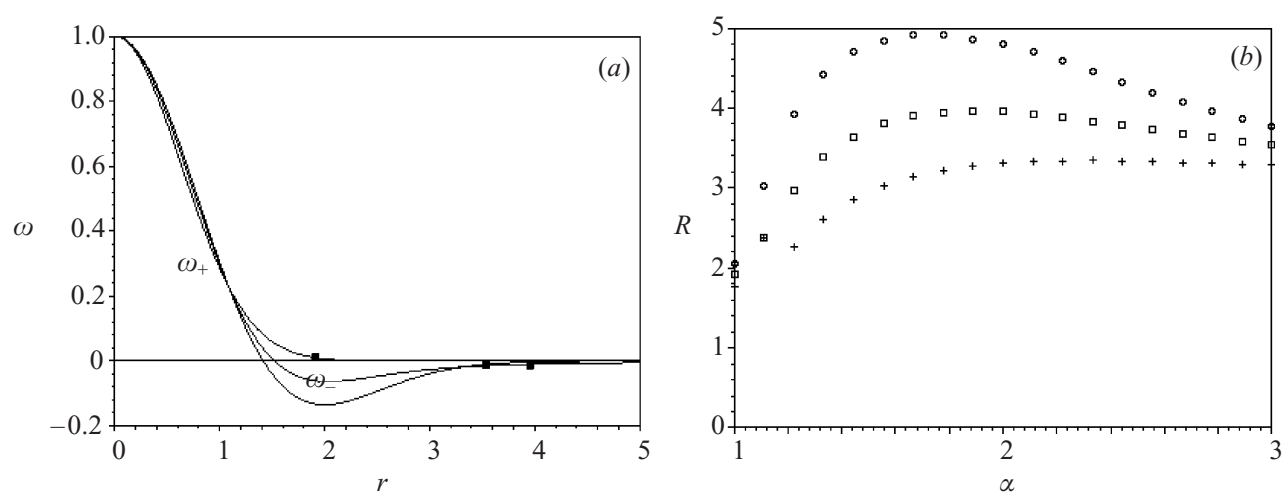

Figure 11. (a) $\mathbf{\square}$, The dimensionless vortex radius $R / R_{v m}$ determined from vorticity profiles given by (4.1) with $\alpha=1.0,1.8$ and 3 by the criterion $|\omega|>1 \% \omega_{\max }$. (b) The variation of this radius with $\alpha$ for the criteria; $\square,|\omega|>0.5 \% \omega_{\max } ; O,|\omega|>1 \% \omega_{\max } ;+,|\omega|>2 \% \omega_{\max }$.

characterized by $\alpha \sim 3$ propagate to the north because the lift force is weak whereas vortices characterized by $\alpha \sim 1$ move to the northwest.

\subsection{Comparison between model and experiments}

To interpret the effect of $\alpha$ on the vortex dynamics, the translational speed $U$ and direction of motion were measured for different times after vortex generation $t=0$. The initial flow in the monopolar vortex is azimuthal and described by (4.1) which is known from the analysis in the measurement-section and includes all velocity profiles ranging from isolated to non-isolated vortices. In order to compare the model with the experimental measurements, a vortex radius $R$ is chosen and is defined to be the outer radius where the absolute value of the vorticity has decreased to $1 \%$ of its maximum value. The impact of vorticity on the vortex outside this region is considered to be negligible; the $1 \%$ threshold was chosen because it is comparable to the experimental error in the vorticity measurements. Figure 11( $a$ ) shows three examples of the choice of $R$ for different values of $\alpha(1.0,1.8$ and 3). Figure 11(b) demonstrates the sensitivity of $R$ to the chosen percentage which was $1 \%$. In the range of $1.2<\alpha<2$, the vorticity tends very slowly to zero so that a small percentage may result in a very large radius (see figure 11a); larger percentages show less variation.

The translation velocity and angle of propagation were calculated with (5.8) using the experimental values for $R_{v m}, v_{\theta m}, \beta$ and $\alpha$. Comparison with the experimental data showed best agreement for time $t=t_{*} / \beta R_{v m}$, based on the time scale $1 / \beta R_{v m}$. The dimensionless time $t_{*}$ was used as a fit parameter and best results were obtained for $t_{*}=0.8$. Comparisons between experimental measurements and model predictions are given in figure 12(a) for the translation speed $U$ and the translation direction in figure $12(b)$, with the ideal correlation corresponding to a straight line. The closed symbols represent experiments with $\alpha>1$.4, whereas the square symbols represent sink vortices characterized by $\alpha<1$.4. Sink vortices generally move to the northwest with a faster translational speed than the model predicts (figure $12 a$ ). When $\alpha<1.4$, the difference between the predictions and experimental measurements is due to the dominant influence of Rossby waves on the vortex propagation, which is not included in the model. The error bars indicate the effect of the variation in the choice of the above-mentioned percentage between $0.5 \%$ and $2 \%$ for vortices with $\alpha>1.4$. As was to be expected from figures 11, the deviation becomes larger with decreasing values of $\alpha$. Nevertheless, the agreement between model predictions and experimental 

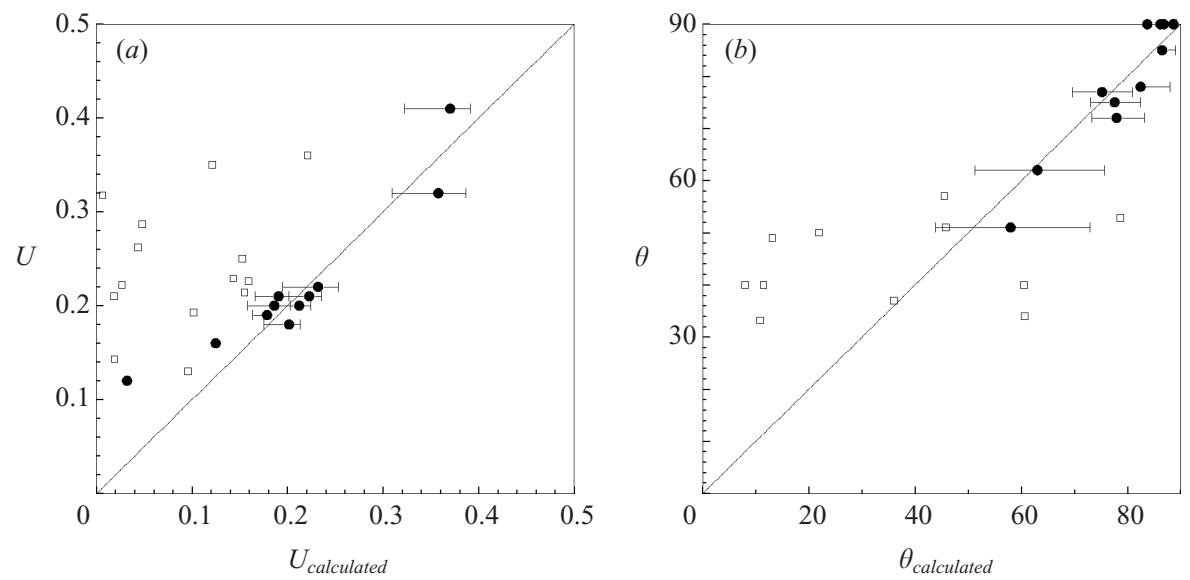

FIGURE 12. Comparison between experimental measurements (vertical axes) and the prediction by the mechanistic model (horizontal axes) obtained from (5.8) for $(a)$ the translation velocity and (b) the direction of propagation. - , values for $\alpha>1.4$; $\square$, values for $\alpha<1.4$. In both graphs the error bars indicate the variation in the predicted values when the criterion for the vortex radius is slightly modified $\left(|\omega|>0.5 \% \omega_{\max }\right.$ to $\left.|\omega|>2 \% \omega_{\max }\right)$. The corresponding variation in radius is shown in figure 11 .

measurement is satisfactory for isolated vortices characterized by $\alpha>1.4$, and shows the difference with the vortices dominated by Rossby wave dynamics.

\section{Discussion}

\subsection{Isolated vortices}

The main difference between the mechanistic model and previous asymptotic models is that the coherence of the vortex is conserved by supposing the vortex as a cylindrical patch during its time evolution. The experimental observations justify this approach: the vortices 'leak' vorticity but they keep their coherence during the initial flow evolution. The mechanistic model simplifies the inherent complex dynamics of monopolar vortices by neglecting the influence of the vorticity generated in the outer field. In fact, this influence is to some extent replaced by the lift force; when radiation by Rossby waves becomes important and the far flow contains vorticity, the model breaks down. Nevertheless, this model shows that the forces acting on a cylindrical patch derived from potential flow theory describe reasonably well the propagation direction and speed of the vortex for isolated vortices (with $1.4<\alpha<3$ ).

Figure 13 shows the direction of motion and translational speed calculated for dimensionless times $t v_{\theta m} / R_{v m}=5,10$ and 20, which correspond to $t=O(30,60)$ and $O(120)$ seconds in the experiments in this paper. The angle of propagation $\theta$ increases monotonically with $\alpha$, showing the same trend as depicted in figure $10 a$. Included in figure 13 are the corresponding theoretical predictions of Reznik \& Dewar (1994). The general description of Reznik \& Dewar (1994) is based on the limit $v_{\theta m} / \beta R_{v m}^{2} \gg 1$ and provides integrable expressions (see Appendix) to calculate the vortex trajectory based on the initial azimuthal velocity profile of the vortex. Both the mechanistic model and the prediction by Reznik \& Dewar (1994) show the same trend for small $\alpha$, whereas for $\alpha>1.5$, the Reznik \& Dewar (1994) model underpredicts the angle of motion which tends to values much smaller than $90^{\circ}$.

Reznik \& Dewar (1994) mention that the energy and angular momentum is infinite 

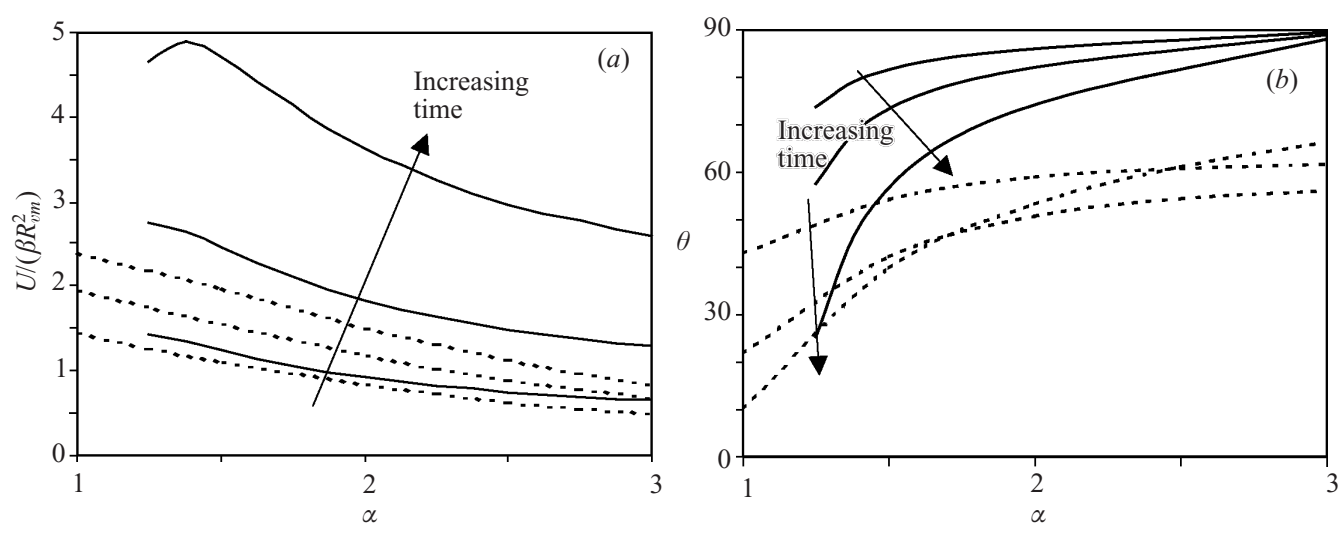

FIGURE 13. Theoretical predictions by $\_$, the mechanistic model and - - - Reznik \& Dewar (1994) of $(a)$ the translational speed of a vortex at times $v_{\theta m} t / R_{m}=5,10$ and 20 and $(b)$ the direction of motion.

in the far field. This implies dominant Rossby waves, and raises important questions about the far-field assumptions. These Rossby waves drive the vortex westward. Reznik \& Dewar results indeed show a tendency of a stronger westward vortex motion than the present experimental results suggest.

\subsection{Non-isolated vortices}

For $\alpha<1.4$, the Rossby waves generated by the slowly decaying velocity field determine the vortex propagation speed which is faster than for the case $\alpha>1.4$.

Korotaev \& Fedotov (1994) studied the motion of an intense Gaussian vortex (i.e. $\alpha=1$ ) propagating on a beta-plane assuming that the vortex moves primarily in a westerly direction. They treated the vortex as a coherent structure, and divided the flow into a fluid region transported with the vortex, enclosed by the stagnation streamline, and the flow outside. Using asymptotic analysis, they showed that the vortex moves to the west relative to the surrounding fluid while being transported to the north by the broad flow induced by Rossby waves. They suggest that their vortex has the same phase (and speed) as the Rossby wave and that the energy of the vortex is communicated into Rossby waves propagating away from the vortex. Though our vortices with $\alpha<1.4$ propagate with approximately half the phase speed, our experimental results support this view qualitatively. However, the direction of propagation of their vortices depends strongly on their small parameter $(\delta)^{-1 / 4}$, an effect that they consider a paradox that may arise from the use of asymptotic series.

Llewellyn Smith (1997) showed that the zeroth-order response to a non-isolated (Gaussian) vortex is a linear Rossby wave with amplitude proportional to the circulation of the vortex. Indeed, the circulation calculated from experimental values on a limited domain of size $10 R_{v m}$ correlated approximately linearly with the vortex translaton speed of vortices characterized by $1.0 \leqslant \alpha \leqslant 1.2$. (The robustness of this result was tested by calculating the circulation over a significantly larger region, $100 R_{v m}$.) For different values of $\delta$, the calculations of Llewellyn Smith show that the vortex centre represented by the relative vorticity maximum moves initially in the same direction $(\theta \approx 70)$ and, depending on the initial value of $\delta$, move sooner or later to the west. The present results show that the vortex velocity and propagation direction are dominated by Rossby waves that transport the vortex in the northwest 
direction between $34^{\circ}$ and $48^{\circ}$. The variation in $\delta$ (from 7 to 110 ; see table 2) reported here did not appear to lead to a variation in propagation direction.

Current asymptotic models appear to predict the vortex translation speed but fail to predict the vortex translation direction. As shown in figure 10, we can predict the translation velocity by using the correct scaling, which is similar in all asymptotic models. However, the direction of propagation is more difficult to predict. The experiments suggest that the vortices (for $\alpha<1.4$ ) are transported by the Rossby waves, implying a different approach from a matching between the vortex edge and ambient of the vortex. The influence of the vortex patch is presumably overestimated by this approach, thus explaining the more northward propagation direction of Gaussian vortices by Llewellyn Smith (1997).

\section{Conclusions}

Based on a systematic experimental study over a wide range of parameters and physical models, we have shown that the initial direction of motion of the vortex depends critically on $\alpha$, which is a measure of the steepness of the velocity profile, and weakly dependent on its original size $R_{v m}$ and maximum azimuthal velocity $v_{\theta m}$. The initial velocity profile within the vortex essentially controls the ratio of the Rossby force, which drives the vortex north, and the lift force which drives the vortex initially west. Compact vortices characterized by $\alpha \sim 3$ initially move to the north because the lift force is weak whereas vortices characterized by $\alpha \sim 1$ initially move to the northwest.

When $\alpha<1.4$, Rossby wave generation appears to dominate the vortex dynamics. Comparison with the long-wave Rossby wave speed, based on a balance between Rossby wave speed and vortex speed, shows a strong correlation with experimental observations (figure $10 b$ ). Generally, the translational speed of the vortex depends on both $\alpha$ and $v_{m \theta} / \beta R_{v m}^{2}$.

For large time, the structure of the vortices changes, but the direction of motion still appeared to be correlated to the instantaneous value of $\alpha$. Isolated vortices $(\alpha \sim 3.0)$ ultimately detrain anticyclonic vorticity so that $\alpha$ decreases and the vortex eventually moves to the northwest. Conversely, intense suction vortices increase in $\alpha$ and showed a tendency to eventually move to the north. In the final stage, Rossby waves were relatively strong and obscured the weak vortex.

To interpret the effect of the initial structure of the vortex (characterized by $\alpha$ ) on the vortex dynamics, a mechanistic model of vortex dynamics was developed by extending Rossby's original model by the inclusion of a lift force. Despite the simplicity of the model, it has a clear physical interpretation with the vortex moving northward owing to the Rossby force and westward owing to the lift force. Favourable comparison with the experimental observations is found. The effect of Rossby wave drag on the vortex translation is not a feature of the model, and comparison of the model with the experimental data distinguishes the two regimes $(\alpha<1.4$ and $\alpha>1.4)$. The general trend of the direction of motion and translational speed with $\alpha>1.4$ is supported by the model.

The correlation between the direction of propagation and the internal structure of the vortex, reported here for $B u \ll 1$, is similar to that reported by Stegner \& Zeitlin for the case of $B u \gg 1$. Stegner \& Zeitlin (1998) correlated $\lambda$, the ratio of the free-surface elevation to the fluid-layer thickness, with the direction of translation $\theta$ and observed $\theta$ to increase with $\lambda^{-1}$. In the present paper, the experiments were characterized by $B u \ll 1$ and the direction of motion $\theta$ increased with the azimuthal 
vorticity profile $(\alpha)$. This suggests that the underlying mechanism which controls the dynamics of vortices may be comparable in the limits of $B u \ll 1$ and $B u \gg 1$, where the relative vorticity in (1.1) plays a similar role to the potential vorticity $(\omega)$ as the free surface $(\eta)$ when $B u \gg 1$.

The transport of pollution by vortices is related to their direction and speed of propagation and was first studied for dipolar vortices on the $f$-plane (Eames \& Flór 1998). The mechanistic model offers new perspectives that enables further quantitative study on the displacement of fluid by vortices moving on the beta-plane which will be subject of a forthcoming investigation.

The authors gratefully acknowledge the help of the Coriolis team for setting up the experiments at the Coriolis platform. I. E. gratefully acknowledges the financial support from the Leverhulme Trust and EPSRC through the award of a Research Fellowship. The collaboration was enabled by the financial assistance from the European Science Foundation.

\section{Appendix}

The asymptotic solution for the vortex trajectory calculated by Reznik \& Dewar (1994) is outlined. The azimuthal velocity is scaled with $v_{\theta m}$ and time scale by $R_{v m} / v_{\theta m}$. The velocity of the vortex centre is calculated

$$
\tilde{v}+\mathrm{i} \tilde{u}=\tilde{t} \int_{0}^{\infty} F(\tilde{r}) \exp \left(-\frac{\mathrm{i} \tilde{v}_{\theta} \tilde{t}}{\tilde{r}}\right) \mathrm{d} \tilde{r},
$$

where $F(\tilde{r})=\left(1 / \tilde{r}^{3}\right) \int_{0}^{\tilde{r}} \tilde{r}^{2} \tilde{v}_{\theta} \mathrm{d} \tilde{r}, \tilde{v}=v_{\theta}(\tilde{r}) / v_{\theta m}$ and $\tilde{r}=r / R_{v m}$. The velocity profile is calculated by rescaling the solution (4.1) with the maximum angular velocity and corresponding radius. The trajectory of the vortex centre is calculated by integrating (A 1) with respect to time.

\section{REFERENCES}

ADEM, J. 1956 A series solution for the barotropic vorticity equation and its application in the study of atmospheric vortices. Tellus 8, 364-372.

Batchelor, G. K. 1967 An Introduction to Fluid Mechanics. Cambridge University Press.

Carnevale, G. F., Kloosterziel, R. C. \& van Heisst, G. J. F. 1991 Propagation of barotropic vortices over topography in a rotating tank. J. Fluid Mech. 233, 119-139.

Carton, X. J., Flierl, G. R. \& Polvani, L. M. 1989 The generation of tripoles from unstable axisymmetric isolated vortex structures. Europhys. Lett. 9, 339-344.

Eames, I. \& Flór, J. B. 1998 Fluid transport by a dipolar vortex. Dyn. Atmos. Oceans 28, 93-100.

Firing, E. \& Beardsley, R. C. 1976 The behavior of a barotropic eddy on a beta-plane. J. Phys. Oceanogr. 6, 57-65.

Flierl, G. R., Larichev, V. D., McWilliams, J. C. \& Reznik, G. M. 1980 The dynamics of baroclinic and barotropic solitary eddies. Dyn. Atmos. Oceans 5, 1-41.

FlóR, J. B. \& van Heisst, G. J. F. 1996 Stable and unstable monopolar vortices in a stratified fluid. J. Fluid Mech. 311, 257-287.

Fincham, A. M. \& Spedding, G. B. 1997 Low cost, high resolution DPIV for measurement of turbulent fluid flow. Exps Fluids 23, 449-462.

Gradshteyn, I. S. \& Ryzhik, I. M. 1980 Tables of Integrals, Series, and Products. Academic Press. van Heisst, G. J. F. 1994 Topographic effects on vortices in a rotating fluid. Meccanica 29, 431-451.

van Heijst, G. J. F., Kloosterziel, R. C. \& Williams, C. W. M. 1991 Laboratory experiments on the tripolar vortex in a rotating fluid. J. Fluid Mech. 225, 301-331. 
Honyi, H., Taneda, S. \& Tatsudo, M. 1980 Some practical details of the electrolytic precipitation method of flow visualization. Rep. Res. Inst. Appl. Mech. 28, 83-89.

Hunt, J. C. R. 1987 Vorticity and vortex dynamics in complex turbulent flows. Trans. Can. Soc. Mech. Engrs 11, 21-35.

Korotaev, G. K. \& Fedotov, A. B. 1994 Dynamics of an isolated barotropic eddy on a beta-plane J. Fluid Mech. 264, 277-301.

LAMB, H. 1932 Hydrodynamics, 6th edn.

Llewellyn SMith, S. G. 1997 The motion of a non-isolated vortex on the beta-plane. J. Fluid Mech. 346, 149-179.

McWilliams, J. C. \& Flierl, G. C. 1979 On the evolution of isolated, nonlinear vortices. J. Phys. Oceanogr. 9, 1155-1182.

Meleshio, V. V. \& van Heisst, G. J. F. 1994 On Chaplygin's investigations of two-dimensional vortex structures in an inviscid fluid. J. Fluid Mech. 272, 157-182.

Masuda, A., Marubayashi, K. \& Ishibashi, M. 1990 A laboratory experiment and numerical simulation of an isolated barotropic eddy in a basin with topographic $\beta$ plane. J. Fluid Mech. 213, 641-655.

Pedlosky, J. 1979 Ocean Circulation Theory. Springer.

RezNik, G. M. 1992 Dynamics of singular vortices on a beta-plane. J. Fluid Mech. 240, 405-432.

REZnIK, G. M. \& Dewar, W. K. 1994 An analytical theory of distributed axisymmetric barotropic vortices on the beta-plane. J. Fluid Mech. 269, 301-321.

Rossby, C.-G. 1948 On displacements and intensity changes of atmospheric vortices. J. Mar. Res. 175-187.

Stegner, A. \& Zeitlin, V. 1998 From quasi-geostrophic to strongly non-linear monopolar vortices in a paraboloidal shallow-water-layer experiment. J. Fluid Mech. 356, 1-24.

Stern, M. E. \& RADKo, T. 1998 The self-propagating quasi-monopolar vortex. J. Phys. Oceanogr. 28, 22-39.

SutYrin, G. G. \& Flierl, G. R. 1994 Intense vortex motion on the beta-plane - development of the beta gyres. J. Atmos. Sci. 51, 773-790.

Velasco Fuentes, O. U. \& van Heisst, G. J. F. 1994 Experimental study of dipolar vortices on a topographic beta-plane. J. Fluid Mech. 259, 79-106. 